JOURNAL OF THE AMERICAN MATHEMATICAL SOCIETY

Volume 20, Number 1, January 2007, Pages 185-210

S 0894-0347(06)00547-9

Article electronically published on August 28, 2006

\title{
BOUNDARY BEHAVIOR OF SLE
}

\author{
NAM-GYU KANG
}

\section{INTRODUCTION AND RESULTS}

Introduction. Several lattice models from statistical physics such as random walks (RWs), loop-erased random walks (LERWs), self-avoiding random walks (SAWs), and critical FK (Fortuin and Kasteleyn) percolations have been shown or are conjectured to be invariant under conformal mappings. The stochastic Loewner evolution (SLE) was first introduced by O. Schramm as a possible scaling limit for the planar LERW [33. As a one-parameter family of random growth processes, the SLE curves are the only random non-self-crossing curves with a certain Markovian type property and conformal invariance.

Loewner chains are widely applied in complex analysis. For instance, de Branges used Loewner evolutions to prove the Bieberbach conjecture, which states that the $n$th coefficient in the power series of a univalent function in the class $\mathcal{S}$ should be no greater than $n$ [6]. In fact, Loewner introduced this concept in the 1920's in order to calculate an estimate on the third coefficient.

G. F. Lawler, O. Schramm, and W. Werner used SLE 6 to determine the two-sided disconnection exponent for Brownian motion. It led to the proof of Mandelbrot's conjecture that the Hausdorff dimension of the planar Brownian frontier is $4 / 3$. See 21, 22, 23, 24, and 25. The planar Brownian frontier is defined as the boundary of the unbounded component of the complement of the planar Brownian path. In [19, G. F. Lawler expressed the Hausdorff dimension of the planar Brownian frontier in terms of the two-sided disconnection exponent.

Numerous discrete models have been proven or are expected to correspond to $\mathrm{SLE}_{\kappa}$ for some $\kappa$. Using Cardy's formula in Carleson's form, Smirnov proved that the critical site percolation on the triangular grid has a conformal invariant scaling limit. He also showed that the scaling limit is described by SLE 6 [35. G. F. Lawler, O. Schramm, and W. Werner proved that the scaling limits of LERW and the uniform spanning tree (UST) Peano curve with appropriate boundary conditions are, respectively, $\mathrm{SLE}_{2}$ and $\mathrm{SLE}_{8}$ [26. G. F. Lawler, O. Schramm, and W. Werner also showed that if the scaling limit of planar SAWs exists and is conformally invariant, then it is $\mathrm{SLE}_{8 / 3}$ [27]. R. Kenyon conjectured that the scaling limit of

Received by the editors January 31, 2005.

2000 Mathematics Subject Classification. Primary 30C45, 60K35; Secondary 28A80, 60J65.

Key words and phrases. SLE, Schwarzian derivatives, Hölder continuity, Duplantier's duality conjecture.

This research was partially conducted during the period when the author was employed by the Clay Mathematical Institute as a Liftoff Fellow. The author is partially supported by NSF grant DMS 05-05751. 

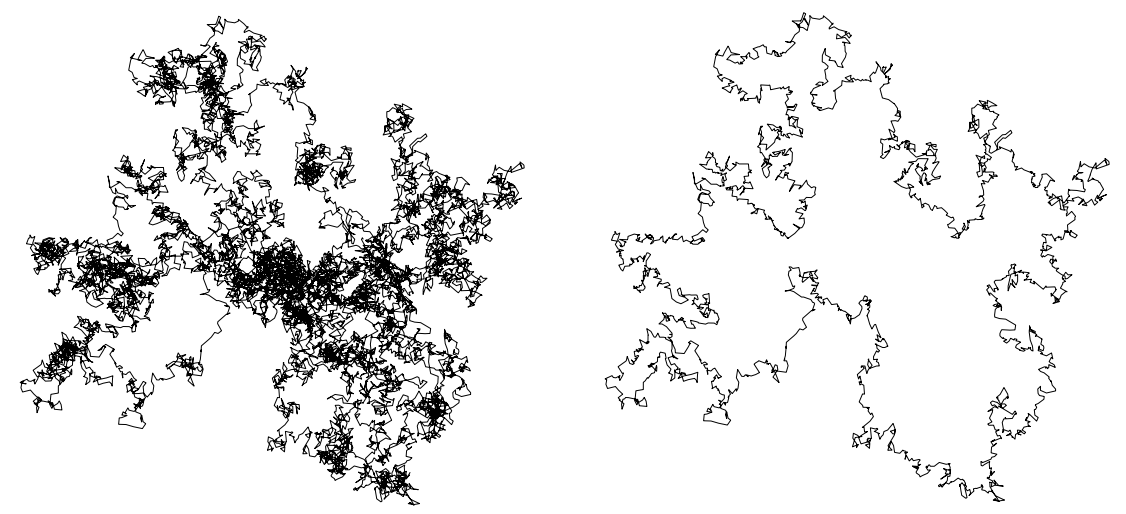

Figure 1. Planar Brownian motion and its frontier.

the double domino paths is $\mathrm{SLE}_{4}$. On the other hand, O. Schramm and S. Sheffield recently proved that the harmonic explorer converges to $\mathrm{SLE}_{4} 34$.

From the dimension estimate for the trace and outer boundary of the hull, B. Duplantier conjectured that $\operatorname{SLE}_{\kappa^{\prime}}\left(\kappa^{\prime}=16 / \kappa\right)$ should describe the boundary of the hull of $\mathrm{SLE}_{\kappa}$ when $\kappa>4$ (see [7]). In particular, the central charge $c$ (see [8] for the definition) of SLE is invariant under the map $\kappa \mapsto 16 / \kappa$ :

$$
c=1-6\left(\sqrt{\frac{\kappa}{4}}-\sqrt{\frac{4}{\kappa}}\right)^{2} .
$$

Duplantier duality has been shown to hold for $\kappa=8$ and $\kappa=6$. In the case $\kappa=8$, the frontier of the UST Peano curve consists of two LERWs, one in the tree and the other in the dual tree. When $\kappa=6$, the restriction property makes it possible to describe the outer boundary of conditioned $\mathrm{SLE}_{6}$ in terms of $\mathrm{SLE}_{8 / 3}$. Using arguments of conformal field theory, B. Duplantier and I. Binder derived the mixed multifractal spectrum $f_{\text {mixed }}(\alpha, \lambda)$ for the scaling and winding (with respect to harmonic measure) in terms of the central charge:

$$
f_{\text {mixed }}(\alpha, \lambda)=\alpha+b-\frac{b \alpha^{2}}{2 \alpha-1-\lambda^{2}},
$$

where $b=(25-c) / 12$. See 2 for definitions and 9 for more details of the results.

Definitions. For each $\kappa \geq 0$ and each $z \in \mathbb{H}$, let $g_{t}(z)$ be the solution of the chordal Loewner equation

$$
\partial_{t} g_{t}(z)=\frac{2}{g_{t}(z)-\sqrt{\kappa} B_{t}}, \quad g_{0}(z)=z,
$$

where $B_{t}$ is a one-dimensional standard Brownian motion on the real line, starting from 0 . The solution exists whenever $g_{t}(z)-\sqrt{\kappa} B_{t}$ is bounded away from zero. This implies $g_{t}(z)$ is well-defined up to the first time $\tau(z)$ such that $\lim _{t \uparrow \tau(z)} g_{t}(z)-$ $\sqrt{\kappa} B_{t}=0$. For each $t>0$, the map $g_{t}$ is a conformal mapping from the domain $H_{t}:=\{z \in \mathbb{H}: \tau(z)>t\}$ onto $\mathbb{H}$. The process $t \mapsto g_{t}$ is called chordal stochastic Loewner evolution in $\mathbb{H}$ with parameter $\kappa$, or $\operatorname{SLE}_{\kappa}$. The sets $K_{t}:=\{z \in \overline{\mathbb{H}}$ : $\tau(z) \leq t\}$ are called the hulls of the SLE. Chordal $\mathrm{SLE}_{\kappa}$ is scale invariant in the following sense. For $c>0$, the process $t \mapsto c^{-1 / 2} K_{c t}$ has the same law as $t \mapsto K_{t}$. The process $(t, z) \mapsto c^{-1 / 2} g_{c t}(\sqrt{c} z)$ has the same law as the process $(t, z) \mapsto g_{t}(z)$. 

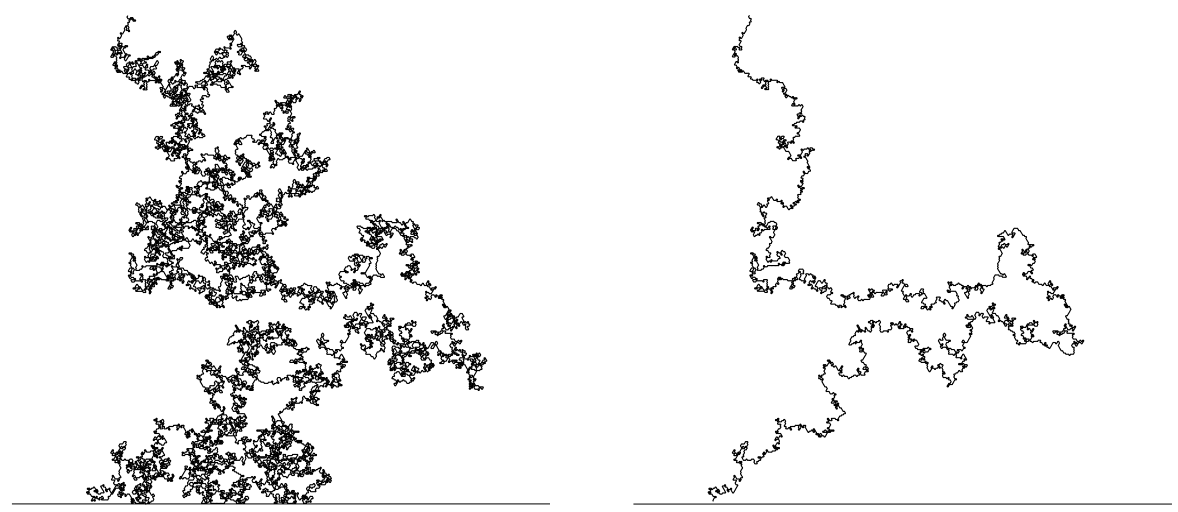

Figure 2. The boundary of the hull of $\mathrm{SLE}_{6}$ describes $\mathrm{SLE}_{8 / 3}$.

There is (almost surely) a uniquely defined continuous path $\gamma:[0, \infty) \rightarrow \overline{\mathbb{H}}$ such that $H_{t}$ is the unbounded component of $\mathbb{H} \backslash \gamma[0, t]$ for all $t \geq 0$. This path is called the $S L E_{\kappa}$ trace from 0 to $\infty$ in $\mathbb{H}$ and is given by

$$
\gamma(t)=\lim _{z \rightarrow 0} g_{t}^{-1}\left(z+\sqrt{\kappa} B_{t}\right) .
$$

Define the backward flow $f_{t}=g_{-t}$ of $g_{t}$ for nonnegative $t$. Then $f_{t}$ is a conformal map from $\mathbb{H}$ into a subset of $\mathbb{H}$ satisfying

$$
\partial_{t} f_{t}(z)=\frac{-2}{f_{t}(z)+\sqrt{\kappa} B_{t}}, \quad f_{0}(z)=z .
$$

For each fixed $t \in \mathbb{R}$, the map $f_{t}(z)$ has the same distribution as the map $z \mapsto$ $g_{t}^{-1}\left(z+\sqrt{\kappa} B_{t}\right)-\sqrt{\kappa} B_{t}$. This follows from the Markov property and translation invariance of Brownian motion. Note that $f_{t}(z)$ is well-defined on $\mathbb{H}$ for all $t \geq 0$.

Basic properties. In 31, S. Rohde and O. Schramm showed that the $\mathrm{SLE}_{\kappa}$ trace is almost surely a continuous path for $\kappa \neq 8$. In the special case $\kappa=8$, this was shown as a consequence of the theorem that the scaling limit of UST Peano curve is chordal $\mathrm{SLE}_{8}$ 26. They estimated the derivative expectations for the backward flow to show that $g_{t}^{-1}$ is almost surely Hölder continuous unless $\kappa=4$ (when it is not Hölder continuous).

V. Beffara established that the Hausdorff dimension of the $\mathrm{SLE}_{\kappa}$ trace is almost surely $\min (1+\kappa / 8,2)[1$. S. Rohde and O. Schramm estimated the convergence exponent for the Whitney decomposition of $H_{1}$ to obtain an upper bound for the box-counting dimension of the boundary of the $\mathrm{SLE}_{\kappa}$ hull. They proved that the Hausdorff dimension of $\partial K_{1}$ is almost surely at most $1+2 / \kappa$ for $\kappa>4$. On the other hand, R. Kenyon conjectured that the Hausdorff dimension of $\partial K_{1}$ is almost surely $1+2 / \kappa$ for $\kappa \geq 4$. (As remarked earlier, this is known for $\kappa=6,8$.) For more basic properties of SLE, see [31, 20], and [37].

(Pre-)Schwarzian derivatives. The logarithmic derivative or pre-Schwarzian derivative $L f$ of a locally univalent function $f$ is defined by

$$
L f(z)=\frac{f^{\prime \prime}(z)}{f^{\prime}(z)} .
$$


The Schwarzian derivative $S f$ of a locally univalent function $f$ is defined by

$$
S f(z)=\left(\frac{f^{\prime \prime}(z)}{f^{\prime}(z)}\right)^{\prime}-\frac{1}{2}\left(\frac{f^{\prime \prime}(z)}{f^{\prime}(z)}\right)^{2} .
$$

The logarithmic derivative and the Schwarzian derivative satisfy the composition law

$$
\begin{aligned}
& L(f \circ g)(z)=L g(z)+L f(g(z)) \cdot g^{\prime}(z), \\
& S(f \circ g)(z)=S g(z)+S f(g(z)) \cdot g^{\prime}(z)^{2} .
\end{aligned}
$$

Thus, the Schwarzian derivative is Möbius-invariant; that is, $S(T \circ f)=S f$ for any Möbius transformation $T$. Also, note that $S T=0$ if and only if $T$ is a Möbius transformation. Suppose $f$ maps $\mathbb{D}$ conformally into $\mathbb{C}$. Then, by the distortion theorem,

$$
\left|\left(1-|z|^{2}\right) L f(z)-2 \bar{z}\right| \leq 4 \quad \text { and } \quad\left(1-|z|^{2}\right)^{2}|S f(z)| \leq 6,
$$

for $z \in \mathbb{D}$. See p. 9 and p. 13 in 29 for the first estimate and the second estimate, respectively. On $\mathbb{H}$, the second estimate in (1.5) becomes

$$
y^{2}|S f(z)| \leq \frac{3}{2},
$$

where $y=\operatorname{Im} z$.

Some analytic criteria for univalence have limited applications because they are far from necessary. The following general criteria involving the (pre-)Schwarzian derivative are useful sufficient conditions and almost necessary in a certain sense. See [29] for more references.

Theorem (Becker univalence criterion). Suppose $f$ is analytic and locally univalent in $\mathbb{D}$. If

$$
\left(1-|z|^{2}\right)|z L f(z)| \leq 1
$$

for $z \in \mathbb{D}$, then $f$ is univalent in $\mathbb{D}$. The bound 1 is sharp.

Theorem (Nehari univalence criterion). Suppose $f$ is analytic and locally univalent in $\mathbb{D}$. If

$$
\left(1-|z|^{2}\right)^{2}|S f(z)| \leq 2
$$

for $z \in \mathbb{D}$, then $f$ is univalent in $\mathbb{D}$. The bound 2 is sharp.

These univalence criteria have the following geometric application: If either estimate (1.6) or (1.7) is uniformly bounded away from the sharp constant, then $f$ maps $\mathbb{D}$ conformally onto a quasi-disk. A Jordan curve $J$ is called a quasi-circle if

$$
\operatorname{diam} J(a, b) \leq C|a-b| \text { for } a, b \in J,
$$

where $J(a, b)$ is the smallest arc (in the sense of diameter) of $J$ between $a$ and $b$. The inner domain of a quasi-circle is called a quasi-disk. The asymptotic behavior of the (pre-)Schwarzian derivative measures the degree of non-conformality of the boundary 29 . 
Beta numbers. In order to study the subsets of rectifiable curves, given a set $E$ in the complex plane and a square $Q, \mathrm{P}$. W. Jones introduced an $L^{\infty}$ version of the beta number

$$
\beta(Q)=\beta_{E}(Q):=\frac{1}{\ell(Q)} \inf _{L \in \mathcal{L}} \sup _{z \in E \cap 3 Q} \operatorname{dist}(z, L),
$$

where $\mathcal{L}$ is the set of all lines $L$ intersecting $Q$ and an $L^{\infty}$ Jones content

$$
\mathcal{J}_{\infty}(E)=\sum_{Q \in \mathcal{D}} \beta_{\infty}^{2}(Q) \ell(Q),
$$

where the summation extends over all dyadic squares.

Theorem A (Jones [17]). Suppose $E$ is a subset of $\mathbb{R}^{2}$. Then $E$ is contained in a rectifiable curve if and only if $\operatorname{diam}(E)$ and the $L^{\infty}$ Jones content $\mathcal{J}_{\infty}(E)$ are finite.

A connected set $E$ is called uniformly wiggly with constant $\beta_{0}$ if $\beta_{E}(Q)>\beta_{0}$ for every $Q$ such that $3^{-1} Q$ intersects $E$ and $\ell(Q) \leq \operatorname{diam}(E)$.

Theorem B (Bishop, Jones 4). Suppose $E \subset \mathbb{R}^{2}$ is a closed, connected, uniformly wiggly set with constant $\beta_{0}$. Then $\operatorname{dim}(E) \geq 1+C \beta_{0}^{2}$, where $C$ is an absolute constant.

C. J. Bishop, P. W. Jones, R. Pemantle, and Y. Peres used a stochastic version of this theorem to prove that the dimension of the Brownian frontier is greater than 1 [5. Also, C. J. Bishop and P. W. Jones showed that large Schwarzian implies large beta numbers. For the precise statement, see [3] or [13. Here, we state the quasiconformal version. Using this, J. Graczyk and P. W. Jones proved that every subarc of the boundary of the Siegel disk (for rotation numbers of "constant type") has the Hausdorff dimension strictly larger than 1 [15. A Jordan curve $\Gamma$ is $K$-quasiconformal (or a $K$-quasicircle) if it is the image of the unit circle by a $K$-quasiconformal homeomorphism $h: \mathbb{C} \rightarrow \mathbb{C}$.

Theorem C (Graczyk, Jones [15]). Suppose that $\Gamma$ is a K-quasicircle. Assume also that there exist $\Delta>0$ and $\epsilon>0$ so that for every $z_{0} \in \mathbb{D}$,

$$
\sup _{\rho\left(z, z_{0}\right) \leq \Delta}|S(h) \| 1-| z||^{2} \geq \epsilon .
$$

Then there exists $\epsilon_{0}>0$ which depends solely on $K, \Delta$, and $\epsilon$ so that

$$
\beta_{\Gamma}>\epsilon_{0} .
$$

Itô's formula. If $X$ is a continuous local martingale and $f$ has two continuous derivatives, then almost surely

$$
f\left(X_{t}\right)-f\left(X_{0}\right)=\int_{0}^{t} f^{\prime}\left(X_{s}\right) d X_{s}+\frac{1}{2} \int_{0}^{t} f^{\prime \prime}\left(X_{s}\right) d\langle X\rangle_{s},
$$

where $\langle X\rangle_{t}$ is the quadratic variation or the variance process of $X_{t}$, which is defined to be the unique continuous process such that $X_{t}^{2}-\langle X\rangle_{t}$ is a local martingale. See [10] or [18. If $X$ and $Y$ are continuous local martingales, then almost surely

$$
X_{t} Y_{t}-X_{0} Y_{0}=\int_{0}^{t} Y_{s} d X_{s}+\int_{0}^{t} X_{s} d Y_{s}+\langle X, Y\rangle_{t}
$$


where $\langle X, Y\rangle_{t}$ is the covariance process of $X$ and $Y$, defined by

$$
\langle X, Y\rangle_{t}=\frac{1}{4}\left(\langle X+Y\rangle_{t}-\langle X-Y\rangle_{t}\right) .
$$

Goluzin's identities. We will use a chordal version of Goluzin's identities to compute the second moment of the (pre-)Schwarzian. Goluzin used a radial version of the identities to obtain the sharpened forms of certain inequalities which constitute the distortion theorems for the class $\Sigma$, consisting of all univalent functions

$$
g(\zeta)=\zeta+b_{0}+b_{1} \zeta^{-1}+\cdots \quad(|\zeta|>1)
$$

in $\widehat{\mathbb{C}} \backslash \overline{\mathbb{D}}$. Suppose $f_{t}$ is a radial Loewner chain:

$$
\frac{d f_{t}}{d t}(z)=-f_{t}(z) \frac{1+k(t) f_{t}(z)}{1-k(t) f_{t}(z)}
$$

where $k(t)$ is a driving function $(|k(t)|=1)$. Goluzin's identities state

$$
\frac{d}{d t} \log \left[\frac{e^{-t}}{f_{t}(z) f_{t}(w)} \frac{f_{t}(z)-f_{t}(w)}{z-w}\right]=-2 \frac{k(t) f_{t}(z)}{1-k(t) f_{t}(z)} \frac{k(t) f_{t}(w)}{1-k(t) f_{t}(w)}
$$

and

$$
\frac{d}{d t} \log \left(1-f_{t}(z) \overline{f_{t}(w)}\right)=2 \frac{k(t) f_{t}(z)}{1-k(t) f_{t}(z)} \overline{\left(\frac{k(t) f_{t}(w)}{1-k(t) f_{t}(w)}\right)} .
$$

See p. 118 in [14. We will state and prove a chordal version of Goluzin's identities in the proof of Lemma 2.1. For the reader's convenience, we give a proof of (1.11) and (1.12).

Proof of Goluzin's identities. It follows from (1.10) that

$$
\begin{aligned}
\frac{d}{d t} \log [ & \left.\frac{e^{-t}}{f_{t}(z) f_{t}(w)} \frac{f_{t}(z)-f_{t}(w)}{z-w}\right]=-1+\frac{d}{d t} \log \left(\frac{1}{f_{t}(w)}-\frac{1}{f_{t}(z)}\right) \\
= & -1+\frac{\frac{1}{f_{t}(w)} \frac{1+k(t) f_{t}(w)}{1-k(t) f_{t}(w)}-\frac{1}{f_{t}(z)} \frac{1+k(t) f_{t}(z)}{1-k(t) f_{t}(z)}}{\frac{1}{f_{t}(w)}-\frac{1}{f_{t}(z)}} \\
= & \frac{\frac{1}{f_{t}(w)}\left(-1+\frac{1+k(t) f_{t}(w)}{1-k(t) f_{t}(w)}\right)-\frac{1}{f_{t}(z)}\left(-1+\frac{1+k(t) f_{t}(z)}{1-k(t) f_{t}(z)}\right)}{\frac{1}{f_{t}(w)}-\frac{1}{f_{t}(z)}} \\
= & \left(\frac{1}{f_{t}(w)}-\frac{1}{f_{t}(z)}\right)^{-1}\left(\frac{2 k(t)}{1-k(t) f_{t}(w)}-\frac{2 k(t)}{1-k(t) f_{t}(z)}\right) \\
= & \frac{f_{t}(z) f_{t}(w)}{f_{t}(z)-f_{t}(w)} \frac{-2 k(t)^{2}\left(f_{t}(z)-f_{t}(w)\right)}{\left(1-k(t) f_{t}(z)\right)\left(1-k(t) f_{t}(w)\right)} \\
= & \frac{-2 k(t)^{2} f_{t}(z) f_{t}(w)}{\left(1-k(t) f_{t}(z)\right)\left(1-k(t) f_{t}(w)\right)} .
\end{aligned}
$$


In a similar way, we obtain

$$
\begin{aligned}
\frac{d}{d t} \log \left(1-f_{t}(z) \overline{f_{t}(w)}\right) & =\frac{-1}{1-f_{t}(z) \overline{f_{t}(w)}}\left(\frac{d f_{t}}{d t}(z) \overline{f_{t}(w)}+\frac{\overline{d f_{t}}(w)}{d t} f_{t}(z)\right) \\
& =\frac{f_{t}(z) \overline{f_{t}(w)}}{1-f_{t}(z) \overline{f_{t}(w)}}\left(\frac{1+k(t) f_{t}(z)}{1-k(t) f_{t}(z)}+\frac{1+\overline{k(t) f_{t}(w)}}{1-\overline{k(t) f_{t}(w)}}\right) \\
& =\frac{2 f_{t}(z) \overline{f_{t}(w)}}{1-f_{t}(z) \overline{f_{t}(w)}} \frac{1-|k(t)|^{2} f_{t}(z) \overline{f_{t}(w)}}{\left(1-k(t) f_{t}(z)\right)\left(1-\overline{k(t) f_{t}(w)}\right)} \\
& =\frac{2 f_{t}(z) \overline{f_{t}(w)}}{\left(1-k(t) f_{t}(z)\right)\left(1-\overline{k(t) f_{t}(w)}\right)}
\end{aligned}
$$

which completes the proof.

Definition of $\mathcal{B M O}$ and the John-Nirenberg inequality. Just as the Hardy space $H^{1}$ is an appropriate substitute for $L^{1}$ in many results concerning singular integrals, the BMO space, or the space of functions of bounded mean oscillation, is a natural substitute for $L^{\infty}$. The BMO space on $\mathbb{R}^{n}$ is the set of equivalence classes of locally integrable functions $f$ (modulo additive constants) for which the inequality

$$
\frac{1}{|Q|} \int_{Q}\left|f(x)-f_{Q}\right| d x \leq C
$$

holds for all cubes $Q$. Here, $f_{Q}$ denotes the mean value of $f$ over the cube $Q$. The smallest such $C$ is taken to be BMO norm and is denoted by $\|f\|_{\text {BMO }}$ (see [36]).

Theorem (John and Nirenberg [16]). There exist positive constants $c$ and $C$ such that for each cube $Q$, each $f \in \mathrm{BMO}$, and each $\lambda$,

$$
\left|\left\{x \in Q:\left|f(x)-f_{Q}\right|>\lambda\right\}\right| \leq C|Q| \exp \left(-c \lambda /\|f\|_{\text {BMO }}\right) .
$$

This notion can be modified in the setting of continuous martingales. A continuous martingale $M$ belongs to the space $\mathcal{B M O}$ of martingales of bounded mean oscillation if there exists $C$ such that for all stopping times $\tau$

$$
\mathbb{E}\left(\left|M_{\infty}-M_{\tau}\right|^{2} \mid \mathcal{F}_{\tau}\right) \leq C^{2}, \quad \text { almost surely. }
$$

The smallest such $C$ is defined as the $\mathcal{B M O}$ norm of $M$ and is denoted by $\|M\|_{\mathcal{B M O}}$. Let $M^{*}=\sup _{t}\left|M_{t}\right|$. For the following results, see pp. 208-211 in [10].

Theorem (Probabilistic analogue of John and Nirenberg inequality). There exists a positive constant $C$ such that for each $M \in \mathcal{B M O}$,

$$
\mathbb{P}\left[M^{*}>\lambda\|M\|_{\mathcal{B M O}}\right] \leq C e^{-\lambda / e} .
$$

Main results. For the SLE related maps, it is well known that $\operatorname{Im} f_{t}(z)$ is monotone increasing in $t$ for every $z \in \mathbb{H}$. For $z \in \mathbb{H}$ and $u \in \mathbb{R}$, set a stopping time

$$
T_{u}=T_{u}(z):=\inf \left\{t \in \mathbb{R}: \operatorname{Im}\left(f_{t}(z)\right) \geq e^{u}\right\} .
$$

It is also well known that for all $z \in \mathbb{H}$, almost surely $T_{u} \neq \infty$. See [31.

Theorem 1. For any $u \in \mathbb{R}$ and $x \neq 0$, the normalized pre-Schwarzian $y L f_{t}(x+i y)$ at $t=T_{u}$ has the asymptotic second moment

$$
\lim _{y \rightarrow 0} \mathbb{E}\left[\left|y L f_{T_{u}}(x+i y)\right|^{2}\right]=\frac{1}{2(\sqrt{\kappa / 4}+\sqrt{4 / \kappa})^{2}} .
$$


Furthermore, for fixed $z=x+i y(x \neq 0) \in \mathbb{H}$,

$$
\lim _{t \rightarrow \infty} \mathbb{E}\left[\left|y L f_{t}(z)\right|^{2}\right]=\frac{1}{2(\sqrt{\kappa / 4}+\sqrt{4 / \kappa})^{2}} .
$$

The normalized pre-Schwarzian $y L f_{t}(z)$, after we subtract a negligible term, is a complex martingale of $\mathcal{B M O}$. To see this, define a random conformal map $F_{t}$ from $\mathbb{H}$ into a subset of $\mathbb{C} \backslash \mathbb{R}_{+}$by $F_{t}(z)=\left(f_{t}(z)+\sqrt{\kappa} B_{t}\right)^{2}$ and set

$$
\begin{aligned}
L_{t}=L_{t}(z) & :=\frac{\kappa}{4+\kappa} y L f_{t}(z)+\frac{4}{4+\kappa}\left(y L F_{t}(z)-y L F_{0}(z)\right) \\
& =y L f_{t}(z)+\frac{4}{4+\kappa}\left(\frac{y f_{t}^{\prime}(z)}{f_{t}(z)+\sqrt{\kappa} B_{t}}-\frac{y}{z}\right) \\
& =\frac{-2}{\sqrt{\kappa / 4}+\sqrt{4 / \kappa}} \int_{0}^{t} \frac{y f_{s}^{\prime}(z)}{\left(f_{s}(z)+\sqrt{\kappa} B_{s}\right)^{2}} d B_{s} .
\end{aligned}
$$

Theorem 2. The process $L$ is a $\mathcal{B M O}$ martingale in $t$ and

$$
\|L\|_{\mathcal{B M O}}^{2}=\frac{1}{2(\sqrt{\kappa / 4}+\sqrt{4 / \kappa})^{2}} .
$$

Theorem 3. For any $u \in \mathbb{R}$ and $x \neq 0$, the normalized Schwarzian $y^{2} S f_{t}(x+i y)$ at $t=T_{u}$ has the asymptotic second moment

$$
\lim _{y \rightarrow 0} \mathbb{E}\left[\left|y^{2} S f_{T_{u}}(x+i y)\right|^{2}\right]=\frac{9 \kappa}{(\kappa+6)(3 \kappa+8)} .
$$

Furthermore, for $x \neq 0$, we have

$$
\lim _{y \rightarrow 0}\left(\lim _{t \rightarrow \infty} \mathbb{E}\left[\left|y^{2} S f_{t}(x+i y)\right|^{2}\right]\right)=\frac{9 \kappa}{(\kappa+6)(3 \kappa+8)} .
$$

The normalized Schwarzian $y^{2} S f_{t}(z)$, after we subtract a negligible term, is a complex martingale of $\mathcal{B M O}$. To see this, set

$$
\begin{aligned}
S_{t}=S_{t}(z) & :=\frac{3 \kappa}{3 \kappa+8} y^{2} S f_{t}(z)+\frac{8}{3 \kappa+8}\left(y^{2} S F_{t}(z)-y^{2} S F_{0}(z)\right) \\
& =y^{2} S f_{t}(z)+\frac{12}{3 \kappa+8}\left(\frac{y^{2}}{z^{2}}-\frac{y^{2} f_{t}^{\prime}(z)^{2}}{\left(f_{t}(z)+\sqrt{\kappa} B_{t}\right)^{2}}\right) \\
& =\frac{24 \sqrt{\kappa}}{3 \kappa+8} \int_{0}^{t} \frac{y^{2} f_{s}^{\prime}(z)^{2}}{\left(f_{s}(z)+\sqrt{\kappa} B_{s}\right)^{3}} d B_{s} .
\end{aligned}
$$

Theorem 4. The process $S$ is a $\mathcal{B M O}$ martingale in $t$. Furthermore, for $x \neq 0$,

$$
\lim _{y \rightarrow 0}\|S(x+i y)\|_{\mathcal{B M O}}^{2}=\frac{9 \kappa}{(3 \kappa+8)(\kappa+6)} .
$$

Recall the definition of the hyperbolic distance or Poincaré metric $d_{\mathbb{H}}$ :

$$
\cosh d_{\mathbb{H}}\left(z_{1}, z_{2}\right)=1+\frac{\left|z_{1}-z_{2}\right|^{2}}{2 y_{1} y_{2}}=-1+\frac{\left|z_{1}-\overline{z_{2}}\right|^{2}}{2 y_{1} y_{2}},
$$

where $y_{j}=\operatorname{Im} z_{j}(j=1,2)$. See p. 136 in [30]. The normalized (pre-)Schwarzian has correlations that decay exponentially in the hyperbolic distance from $z_{1}=x_{1}+i y_{1}$ to $z_{2}=x_{2}+i y_{2}$. Thus, the normalized (pre-)Schwarzian derivatives are nearly independent if $z_{1}$ and $z_{2}$ are far away from each other. For $z_{1}, z_{2} \in \mathbb{H}$ and $u \in \mathbb{R}$, set a stopping time $T_{u}^{*}=T_{u}^{*}\left(z_{1}, z_{2}\right):=\max \left(T_{u}\left(z_{1}\right), T_{u}\left(z_{2}\right)\right)$. 
Theorem 5. The $\mathcal{B M O}$ martingale $L_{t}$ at $t=\infty$ has exponential decay of correlations:

$$
\left|\mathbb{E} L_{\infty}\left(z_{1}\right) \overline{L_{\infty}\left(z_{2}\right)}\right|=\frac{1}{2(\sqrt{\kappa / 4}+\sqrt{4 / \kappa})^{2}} \cosh ^{-2} \frac{d_{\mathbb{H}}\left(z_{1}, z_{2}\right)}{2} .
$$

Furthermore, given $u \in \mathbb{R}$ and a compact subset $K$ of the real line, $L_{t}$ at $T_{u}^{*}$ has exponential decay of correlations:

$$
\limsup _{y_{1}, y_{2} \rightarrow 0} y_{1} y_{2}\left|\mathbb{E} L_{T_{u}^{*}}\left(z_{1}\right) \overline{L_{T_{u}^{*}}\left(z_{2}\right)}\right| \leq \frac{1}{2(\sqrt{\kappa / 4}+\sqrt{4 / \kappa})^{2}} \cosh ^{-2} \frac{\rho}{2},
$$

where the limsup is taken over all $\left(z_{1}, z_{2}\right) \in(K \times(0,1))^{2}$ such that $d_{\mathbb{H}}\left(z_{1}, z_{2}\right) \geq \rho$.

Theorem 6. The $\mathcal{B M O}$ martingale $S_{t}$ at $t=\infty$ has exponential decay of correlations:

$$
\begin{aligned}
\left|\mathbb{E} S_{\infty}\left(z_{1}\right) \overline{S_{\infty}\left(z_{2}\right)}\right| & \leq \frac{9 \kappa}{(\kappa+6)(3 \kappa+8)} \cosh ^{-4} \frac{d_{\mathbb{H}}\left(z_{1}, z_{2}\right)}{2} \\
& +C_{2}(\kappa) \frac{y_{1} y_{2}}{\left|z_{1} z_{2}\right|} \cosh ^{-2} \frac{d_{\mathbb{H}}\left(z_{1}, z_{2}\right)}{2} .
\end{aligned}
$$

Furthermore, given $u \in \mathbb{R}$ and a compact subset $K(0 \notin K)$ of the real line, $S_{t}$ at $T_{u}^{*}$ has exponential decay of correlations:

$$
\limsup _{y_{1}, y_{2} \rightarrow 0}\left|\mathbb{E} S_{T_{u}^{*}}\left(z_{1}\right) \overline{S_{T_{u}^{*}}\left(z_{2}\right)}\right| \leq \frac{9 \kappa}{(\kappa+6)(3 \kappa+8)} \cosh ^{-4} \frac{\rho}{2},
$$

where the limsup is taken over all $\left(z_{1}, z_{2}\right) \in(K \times(0,1))^{2}$ such that $d_{\mathbb{H}}\left(z_{1}, z_{2}\right) \geq \rho$.

It is likely that this result leads to an estimate on the lower bound for the Hausdorff dimension of the SLE boundary. This should be true because Theorems A, B, C mean that "most often" the boundary is wiggly near $f_{t}(z)$. Furthermore, the decay of correlations from Theorem 6 means that a statistical version of Theorem $\mathrm{C}$ should hold, as it does in [5. The estimate for the upper bound on the Hausdorff dimension is already established by S. Rohde and O. Schramm 31. While the Hausdorff dimension of the $\mathrm{SLE}_{\kappa}$ trace was proved by V. Beffara [1, it remains an open conjecture for the boundary of the hull in case $\kappa>4$.

Computing the derivative expectation or moment generating function for $\left|g_{t}^{\prime}(z)\right|$ for an arbitrary complex number $z, \mathrm{~S}$. Rohde and O. Schramm proved that $g_{t}^{-1}$ is almost surely Hölder continuous when $\kappa \neq 4$ in 31 . We reexamine their derivative expectation to derive the conjectured sharp estimate for the Hölder exponent. After oral communication with us, I. Binder and B. Duplantier derived the same formula from the multifractal spectrum of $\mathrm{SLE}_{\kappa}$ independently.

Theorem 7. Suppose $\kappa \neq 4$, a sufficiently small $c>0$, and a bounded set $D \subset \mathbb{H}$ are given. Then almost surely $f_{1}$ is h-Hölder continuous in $D$ on the event that $\operatorname{Im} f_{1}(z) \geq c$ in $D$, provided

$$
h<h(\kappa):=1-\frac{1}{\mu}-\sqrt{\frac{1}{\mu^{2}}+\frac{2}{\mu}},
$$

where $\mu=\kappa / 4+2+4 / \kappa$. 


\section{2. (PRe-)SchWARZian DERIVATives}

Suppose $f_{t}$ is a general Loewner chain with a driving function $U_{t}$. By direct calculation, we observe

$$
\partial_{t} L f_{t}(z)=\frac{-4 f_{t}^{\prime}(z)}{\left(f_{t}(z)+U_{t}\right)^{3}}, \quad \partial_{t} S f_{t}(z)=\frac{12 f_{t}^{\prime}(z)^{2}}{\left(f_{t}(z)+U_{t}\right)^{4}} .
$$

Consider a Loewner evolution $F_{t}:=\left(f_{t}+U_{t}\right)^{2}$ in the slit domain $\mathbb{C} \backslash \mathbb{R}_{+}$. Suppose a sufficiently small $c>0$ and a bounded set $D \subset \mathbb{H}$ are given. Here, by bounded set we mean bounded in the sense of the Euclidean metric on $\mathbb{R}^{2}$ (not the hyperbolic metric on $\mathbb{H}$ ). Due to the composition law for the (pre-)Schwarzian derivatives, the normalized (pre-)Schwarzian derivatives of $F_{t}$ and $f_{t}$ have the same asymptotic behavior in $D$, as long as $\inf _{z \in D} \operatorname{Im} f_{t}(z) \geq c$. By (1.4), observe

$$
L F_{t}(z)=L f_{t}(z)+\frac{f_{t}^{\prime}(z)}{f_{t}(z)+U_{t}}
$$

and

$$
S F_{t}(z)=S f_{t}(z)-\frac{3}{2}\left(\frac{f_{t}^{\prime}(z)}{f_{t}(z)+U_{t}}\right)^{2} .
$$

For instance, if $\inf _{z \in D} \operatorname{Im} f_{t}(z) \geq c$, then, by the Koebe distortion theorem, we have

$$
\left|y L F_{t}(z)-y L f_{t}(z)\right| \leq \frac{\left|y f_{t}^{\prime}(z)\right|}{\left|f_{t}(z)+U_{t}\right|} \leq \frac{\left|y f_{t}^{\prime}(z)\right|}{c} \leq C \operatorname{dist}\left(f_{t}(z), \partial f_{t}(\mathbb{H})\right) .
$$

Now, consider the SLE backward flow $f_{t}$. Use the Itô formula to obtain

$$
\begin{aligned}
d \frac{f_{t}^{\prime}(z)}{f_{t}(z)+\sqrt{\kappa} B_{t}} & =(\kappa+4) \frac{f_{t}^{\prime}(z)}{\left(f_{t}(z)+\sqrt{\kappa} B_{t}\right)^{3}} d t \\
& -\sqrt{\kappa} \frac{f_{t}^{\prime}(z)}{\left(f_{t}(z)+\sqrt{\kappa} B_{t}\right)^{2}} d B_{t}
\end{aligned}
$$

and

$$
\begin{aligned}
d\left(\frac{f_{t}^{\prime}(z)}{f_{t}(z)+\sqrt{\kappa} B_{t}}\right)^{2} & =(3 \kappa+8) \frac{f_{t}^{\prime}(z)^{2}}{\left(f_{t}(z)+\sqrt{\kappa} B_{t}\right)^{4}} d t \\
& -2 \sqrt{\kappa} \frac{f_{t}^{\prime}(z)^{2}}{\left(f_{t}(z)+\sqrt{\kappa} B_{t}\right)^{3}} d B_{t} .
\end{aligned}
$$

To show (2.5), let $Z_{t}=f_{t}^{\prime}(z)$ and $W_{t}=1 /\left(f_{t}(z)+\sqrt{\kappa} B_{t}\right)$. By the Itô formula, $d Z_{t}=$ $2 Z_{t} W_{t}^{2} d t$ and $d W_{t}=(\kappa+2) W_{t}^{3} d t-\sqrt{\kappa} W_{t}^{2} d B_{t}$. On the other hand, $d\langle Z, W\rangle_{t}=0$. It follows from (1.9) that

$$
d\left(Z_{t} W_{t}\right)=(\kappa+4) Z_{t} W_{t}^{3} d t-\sqrt{\kappa} Z_{t} W_{t}^{2} d B_{t} .
$$

For (2.6), the Itô formula and (2.5) imply that

$$
d\left(Z_{t} W_{t}\right)^{2}=2 Z_{t} W_{t} d\left(Z_{t} W_{t}\right)+d\langle Z W\rangle_{t}=(3 \kappa+8) Z_{t}^{2} W_{t}^{4} d t-2 \sqrt{\kappa} Z_{t}^{2} W_{t}^{3} d B_{t} .
$$

Combining (2.1), (2.2), (2.3), (2.5), and (2.6), one can easily check the identities in (1.17) and (1.20). 


\section{Pre-Schwarzian expectation.}

Lemma 2.1. Suppose $f_{t}$ is a general Loewner chain with a driving function $U_{t}$. Then the total variation of the modified Schwarzian derivative of $f_{t}$ is

$$
y^{2} \int_{0}^{t}\left|\partial_{s} S f_{s}(z)\right| d s=\frac{3}{2}\left(1-\frac{\left|y f_{t}^{\prime}(z)\right|^{2}}{\left(\operatorname{Im} f_{t}(z)\right)^{2}}\right) .
$$

Proof. Based on a radial version, it is not hard to formulate and prove Goluzin's identities in $\mathbb{H}$ :

$$
\frac{d}{d t} \frac{f_{t}^{\prime}(z) f_{t}^{\prime}(w)}{\left(f_{t}(z)-f_{t}(w)\right)^{2}}=\frac{2 f_{t}^{\prime}(z) f_{t}^{\prime}(w)}{\left(f_{t}(z)+U_{t}\right)^{2}\left(f_{t}(w)+U_{t}\right)^{2}}
$$

and

$$
\frac{d}{d t} \frac{f_{t}^{\prime}(z) \overline{f_{t}^{\prime}(w)}}{\left(f_{t}(z)-\overline{f_{t}(w)}\right)^{2}}=\frac{2 f_{t}^{\prime}(z) \overline{f_{t}^{\prime}(w)}}{\left(f_{t}(z)+U_{t}\right)^{2}\left(\overline{f_{t}(w)}+U_{t}\right)^{2}} .
$$

To verify (2.8), we first note that

$$
\frac{d}{d t} \frac{\partial}{\partial z} \frac{\partial}{\partial w} \log \frac{f_{t}(z)-f_{t}(w)}{z-w}=\frac{d}{d t} \frac{f_{t}^{\prime}(z) f_{t}^{\prime}(w)}{\left(f_{t}(z)-f_{t}(w)\right)^{2}} .
$$

On the other hand,

$$
\begin{aligned}
\frac{d}{d t} \frac{\partial}{\partial z} \frac{\partial}{\partial w} \log \frac{f_{t}(z)-f_{t}(w)}{z-w} & =\frac{\partial}{\partial z} \frac{\partial}{\partial w} \frac{2}{\left(f_{t}(z)+U_{t}\right)\left(f_{t}(w)+U_{t}\right)} \\
& =\frac{2 f_{t}^{\prime}(z) f_{t}^{\prime}(w)}{\left(f_{t}(z)+U_{t}\right)^{2}\left(f_{t}(w)+U_{t}\right)^{2}},
\end{aligned}
$$

which shows (2.8). In particular, by letting $w \rightarrow z$ in the above identity,

$$
\frac{d}{d t} \frac{S f_{t}(z)}{6}=\frac{2 f_{t}^{\prime}(z)^{2}}{\left(f_{t}(z)+U_{t}\right)^{4}},
$$

which shows the second part of (2.1). For (2.9), one can use a method similar to that above. Alternately, we compute directly:

$$
\begin{aligned}
\frac{\left(f_{t}(z)-\overline{f_{t}(w)}\right)^{2}}{2 f_{t}^{\prime}(z) \overline{f_{t}^{\prime}(w)}} & \frac{d}{d t} \frac{f_{t}^{\prime}(z) \overline{f_{t}^{\prime}(w)}}{\left(f_{t}(z)-\overline{f_{t}(w)}\right)^{2}} \\
& =\frac{1}{\left(f_{t}(z)+U_{t}\right)^{2}}+\frac{1}{\left(\overline{f_{t}(w)}+U_{t}\right)^{2}} \\
& -\frac{1}{f_{t}(z)-\overline{f_{t}(w)}}\left(\frac{-2}{f_{t}(z)+U_{t}}-\overline{\overline{f_{t}(w)}+U_{t}}\right) \\
& =\frac{\left(\overline{f_{t}(w)}+U_{t}\right)^{2}+\left(f_{t}(z)+U_{t}\right)^{2}-2\left(f_{t}(z)-\overline{f_{t}(w)}\right)}{\left(f_{t}(z)+U_{t}\right)^{2}\left(\overline{f_{t}(w)}+U_{t}\right)^{2}} \\
& =\frac{\left(f_{t}(z)-\overline{f_{t}(w)}\right)^{2}}{\left(\overline{\left.f_{t}(z)+U_{t}\right)^{2}\left(\overline{f_{t}(w)}+U_{t}\right)^{2}} .\right.}
\end{aligned}
$$


It follows from (2.1) and (2.9) that

$$
\begin{aligned}
y^{2} \int_{0}^{t}\left|\frac{d}{d s} S f_{s}(z)\right| d s & =y^{2} \int_{0}^{t} \frac{d}{d s} \frac{6\left|f_{s}^{\prime}(z)\right|^{2}}{\left(f_{s}(z)-\overline{f_{s}(z)}\right)^{2}} d s \\
& =y^{2} \int_{0}^{t} \frac{d}{d s} \frac{6\left|f_{s}^{\prime}(z)\right|^{2}}{-4\left(\operatorname{Im} f_{s}(z)\right)^{2}} d s \\
& =\frac{3}{2}\left(1-\frac{\left|y f_{t}^{\prime}(z)\right|^{2}}{\left(\operatorname{Im} f_{t}(z)\right)^{2}}\right),
\end{aligned}
$$

which completes the proof.

Proof of Theorem 1. By (2.1), (1.17), and the optional stopping theorem, we get

$$
\begin{aligned}
\lim _{y \rightarrow 0} \mathbb{E} \mid y L f_{T_{u}}(z) & +\left.\frac{4}{4+\kappa}\left(\frac{y f_{T_{u}}^{\prime}(z)}{f_{T_{u}}(z)+\sqrt{\kappa} B_{T_{u}}}-\frac{y}{z}\right)\right|^{2} \\
& =\frac{1}{3(\sqrt{\kappa / 4}+\sqrt{4 / \kappa})^{2}} \lim _{y \rightarrow 0} \mathbb{E} \int_{0}^{T_{u}} y^{2}\left|\partial_{s} S f_{s}(z)\right| d s \\
& =\frac{1}{2(\sqrt{\kappa / 4}+\sqrt{4 / \kappa})^{2}}\left(1-\lim _{y \rightarrow 0} \mathbb{E} \frac{\left|y f_{T_{u}}^{\prime}(z)\right|^{2}}{e^{2 u}}\right) .
\end{aligned}
$$

The last equality above comes from Lemma 2.1 and the definition of $T_{u}(1.14)$. We need to prove that $\lim _{y \rightarrow 0} \mathbb{E}\left|y f_{T_{u}}^{\prime}(z)\right|^{2}=0$. By scale invariance, we may assume $u=0$. For $\kappa \neq 4$, it follows from the derivative expectation or Theorem 3.2 in 31 that

$$
\lim _{y \rightarrow 0} \mathbb{E}\left|y f_{T_{0}}^{\prime}(z)\right|^{2} \leq C \lim _{y \rightarrow 0} y^{2\left(2-\frac{1-\sqrt{1-4 / \mu}}{2 / \mu}\right)}=0,
$$

where $\mu=\kappa / 4+2+4 / \kappa$. (This estimate can be found in (4.4) of this paper.) For $\kappa=4$, the Schwarz lemma implies that $\left|y f_{T_{0}}^{\prime}(z)\right| \leq \operatorname{Im} f_{T_{0}}(z)=1$, and hence $\mathbb{E}\left|y f_{T_{0}}^{\prime}(z)\right|^{2} \leq \mathbb{E}\left|y f_{T_{0}}^{\prime}(z)\right|^{3 / 2} \leq C y$. On the other hand,

$$
\lim _{y \rightarrow 0} \mathbb{E}\left|\frac{y f_{T_{u}}^{\prime}(z)}{f_{T_{u}}(z)+\sqrt{\kappa} B_{T_{u}}}\right|^{2} \leq \lim _{y \rightarrow 0} \mathbb{E}\left|\frac{y f_{T_{u}}^{\prime}(z)}{\operatorname{Im} f_{T_{u}}(z)}\right|^{2}=0,
$$

which completes the proof of (1.15).

The second part, the equality (1.16) can be easily proved since $\left|y f_{t}^{\prime}(z)\right| / \operatorname{Im} f_{t}(z)$ tends to 0 as $t \rightarrow \infty$. Indeed, $\operatorname{Im} f_{t}(z)$ is increasing in $t$ and it tends to $\infty$ as $t \rightarrow \infty$. On the other hand, it follows from the Koebe distortion theorem that $\left|y f_{t}^{\prime}(z)\right|$ is comparable to the distance from $f_{t}(z)$ to the boundary $\partial f_{t}(\mathbb{H})$.

In the subsection "BMO norm and ODE" below, we will also prove this second part using a different method.

Pre-Schwarzian and $\mathcal{B M O}$. In a proper setup, the pre-Schwarzian derivatives of $\mathrm{SLE}_{\kappa}$ maps are $\mathcal{B M O}$ martingales. (Recall (1.17).) As a consequence, they satisfy the John-Nirenberg inequality.

Proof of Theorem 2. Suppose a stopping time $\tau$ is given. By the strong Markov property, we have

$$
y^{2} \int_{\tau}^{\infty} \mathbb{E}\left[\left|\partial_{s} S f_{s}(z)\right| \mid \mathcal{F}_{\tau}\right] d s=y^{2}\left|f_{\tau}^{\prime}(z)\right|^{2} \int_{0}^{\infty} \mathbb{E}\left|\partial_{s} S f_{s}\left(f_{\tau}(z)+\sqrt{\kappa} B_{\tau}\right)\right| d s
$$


The Schwarz lemma implies $y\left|f_{\tau}^{\prime}(z)\right| \leq \operatorname{Im}\left(f_{\tau}(z)+\sqrt{\kappa} B_{\tau}\right)$. Therefore, it suffices to show that

$$
y^{2} \mathbb{E} \int_{0}^{\infty}\left|\partial_{s} S f_{s}(z)\right| d s
$$

is uniformly bounded above by a universal constant $C$. (This is just a version of Goluzin's theorem.) Since $\left|y f_{t}^{\prime}(z)\right| / \operatorname{Im} f_{t}(z) \rightarrow 0$ as $t \rightarrow \infty$, it follows from (2.7) that the above integral has the constant value $3 / 2$.

$\mathcal{B M O}$ norm and ODE. The method presented in this subsection turns out to be useful to formulate the Schwarzian expectation. However, the reader may skip this subsection. We will use a different method to prove the second part of Theorem 1

$$
y^{2} \mathbb{E} \int_{0}^{\infty}\left|\partial_{t} S f_{t}(z)\right| d t=\frac{3}{2} .
$$

Claim 1. Define a function $w$ by

$$
w(x)=\frac{1}{6}\left(x^{2}+1\right) \mathbb{E} \int_{0}^{\infty}\left|\partial_{t} S f_{t}(x+i)\right| d t .
$$

Then

$$
y^{2} \mathbb{E} \int_{0}^{\infty}\left|\partial_{t} S f_{t}(z)\right| d t=6 \frac{w(x / y)}{1+(x / y)^{2}} .
$$

Claim 2. The function $w$ satisfies the inhomogeneous $O D E$ : $H w=1$, where $H$ is the second-order linear differential operator given by

$$
H v:=-\frac{\kappa}{4}\left(1+x^{2}\right) v_{x x}+(\kappa+2) x v_{x}-\frac{(4+3 \kappa / 2) x^{2}-(4+\kappa / 2)}{1+x^{2}} v .
$$

Claim 3. $w(x)=\left(x^{2}+1\right) / 4$.

Proof of Claim 1. It follows from the scaling property of SLE that $\mid f_{t}(x+i y)+$ $\sqrt{\kappa} B_{t} \mid$ and $\left|f_{t}^{\prime}(x+i y)\right|$ have the same distribution as $y\left|f_{t y^{-2}}(x / y+i)+\sqrt{\kappa} B_{t y^{-2}}\right|$ and $\left|f_{t y^{-2}}^{\prime}(x / y+i)\right|$, respectively. Consequently, we observe that $\left|\partial_{t} S f_{t}(x+i y)\right|$ and $y^{-4}\left|\left(\partial_{t} S f\right)_{t y^{-2}}(x / y+i)\right|$ are identically distributed. With the substitution $s=t y^{-2}$, we have

$$
y^{2} \mathbb{E} \int_{0}^{\infty}\left|\partial_{t} S f_{t}(x+i y)\right| d t=\mathbb{E} \int_{0}^{\infty}\left|\partial_{s} S f_{s}\left(\frac{x}{y}+i\right)\right| d s,
$$

which completes the proof.

Proof of Claim 2, For $z=x+i \in \mathbb{H}$ and any nonnegative number $u$, we set a stopping time

$$
\tau_{u}=\tau_{u}(z):=\inf \left\{t \geq 0: \operatorname{Im} f_{t}(z) \geq y e^{u}\right\} .
$$

$\tau_{u}$ is well-defined since $\operatorname{Im} f_{t}$ is monotone increasing in $t$. Use a change of variable $u=U_{t}=\log \left(\operatorname{Im} f_{t}(z) / \operatorname{Im} f_{0}(z)\right), d U_{t}=2 /\left|f_{t}(z)+\sqrt{\kappa} B_{t}\right|^{2} d t$ to get

$$
\begin{aligned}
\mathbb{E} \int_{0}^{\infty}\left|\partial_{t} S f_{t}(z)\right| d t & =12 \mathbb{E} \int_{0}^{\infty} \frac{\left|f_{t}^{\prime}(z)\right|^{2} d t}{\left|f_{t}(z)+\sqrt{\kappa} B_{t}\right|^{4}} \\
& =6 \mathbb{E} \int_{0}^{\infty} \frac{\left|f_{\tau_{u}}^{\prime}(z)\right|^{2} d u}{\left|f_{\tau_{u}}(z)+\sqrt{\kappa} B_{\tau_{u}}\right|^{2}} .
\end{aligned}
$$


Let $v(x, s):=\mathbb{E}\left(\left|(x+i) f_{\tau_{s}}^{\prime}(x+i)\right|^{2} /\left|f_{\tau_{s}}(x+i)+\sqrt{\kappa} B_{\tau_{s}}\right|^{2}\right)$. We will show that $v$ is a solution to the parabolic PDE: $v_{s}=-H v$ with an initial condition $v(x, 0)=1$. By (2.5) and the Itô formula, we have

$$
\begin{aligned}
d \log \frac{f_{t}^{\prime}(z)}{f_{t}(z)+\sqrt{\kappa} B_{t}} & =\frac{\left(\frac{1}{2} \kappa+4\right)}{\left(f_{t}(z)+\sqrt{\kappa} B_{t}\right)^{2}} d t \\
& -\frac{\sqrt{\kappa}}{f_{t}(z)+\sqrt{\kappa} B_{t}} d B_{t} .
\end{aligned}
$$

Let $X_{u}=\operatorname{Re}\left(f_{\tau_{u}}(z)+\sqrt{\kappa} B_{\tau_{u}}\right) / \operatorname{Im}\left(f_{\tau_{u}}(z)+\sqrt{\kappa} B_{\tau_{u}}\right)$. Then $X_{u}$ is an Itô diffusion

$$
d X_{u}=-2 X_{u} d u+\sqrt{\frac{\kappa}{2}} \sqrt{1+X_{u}^{2}} d B_{u},
$$

with $X_{0}=x$. Now, $v$ can be expressed as a Feynman-Kac type integral in terms of $X_{u}$ :

$$
v(x, s)=\mathbb{E}\left(\exp \left[\left(4+\frac{\kappa}{2}\right) \int_{0}^{s} \frac{X_{u}^{2}-1}{X_{u}^{2}+1} d u-\sqrt{2 \kappa} \int_{0}^{s} \frac{X_{u}}{\sqrt{X_{u}^{2}+1}} d B_{u}\right]\right) .
$$

Therefore, $v$ satisfies the following parabolic equation with an initial condition:

$$
v_{s}=-H v \text { and } v(x, 0) \equiv 1 .
$$

Here, we use the following version of the Feynman-Kac formula: If $X_{t}$ is an Itô diffusion with

$$
d X_{t}=b\left(X_{t}\right) d t+\sigma\left(X_{t}\right) d B_{t}
$$

then

$$
v=v(x, s)=\mathbb{E}^{x}\left(\exp \left[\int_{0}^{s} p\left(X_{t}\right) d B_{t}-\frac{1}{2} \int_{0}^{s} p\left(X_{t}\right)^{2} d t-\int_{0}^{s} q\left(X_{t}\right) d t\right]\right)
$$

satisfies

$$
v_{s}=\frac{1}{2} \sigma^{2} v_{x x}+(b+p \sigma) v_{x}-q v .
$$

Observe that

$$
w(x)=\int_{0}^{\infty} v(x, t) d t=\int_{0}^{\infty} e^{-H t} v(x, 0) d t=\int_{0}^{\infty} e^{-H t} d t .
$$

Using the formula

$$
(H+\lambda I)^{-1}=\int_{0}^{\infty} e^{-H t} e^{-\lambda t} d t,
$$

we deduce that $w$ is a solution to the inhomogeneous ODE: $H w=1$.

Proof of Claim 3. The function $w$ is of the form

$$
\begin{aligned}
w(x) & =c_{1}\left(1+x^{2}\right)^{\frac{3}{2}+\frac{2}{\kappa}} p_{\nu}^{\mu}(x)+c_{2}\left(1+x^{2}\right)^{\frac{3}{2}+\frac{2}{\kappa}} q_{\nu}^{\mu}(x) \\
& +\left(1+x^{2}\right)^{\frac{3}{2}+\frac{2}{\kappa}} p_{\nu}^{\mu}(x) \int_{x}^{\infty} \frac{4}{\kappa+8} \frac{q_{\nu}^{\mu}(t)}{\left(1+t^{2}\right)^{\frac{3}{2}+\frac{2}{\kappa}}} d t \\
& -\left(1+x^{2}\right)^{\frac{3}{2}+\frac{2}{\kappa}} q_{\nu}^{\mu}(x) \int_{x}^{\infty} \frac{4}{\kappa+8} \frac{p_{\nu}^{\mu}(t)}{\left(1+t^{2}\right)^{\frac{3}{2}+\frac{2}{\kappa}}} d t,
\end{aligned}
$$


where $c_{1}$ and $c_{2}$ are two constants and $p_{\nu}^{\mu}$ and $q_{\nu}^{\mu}$ are two independent solutions to the homogeneous ODE with initial conditions:

$$
\begin{aligned}
& \left(1+x^{2}\right) y^{\prime \prime}+2 x y^{\prime}-\left[\nu(\nu+1)-\mu^{2} /\left(1+x^{2}\right)\right] y=0, \\
& p_{\nu}^{\mu}(0)=0, q_{\nu}^{\mu}(0)=\frac{\Gamma\left(\frac{3}{2}+\nu\right) \Gamma\left(\frac{1}{2}\right)}{\Gamma\left(1+\frac{\mu+\nu}{2}\right) \Gamma\left(1+\frac{-\mu+\nu}{2}\right)}, \\
& \left(p_{\nu}^{\mu}\right)^{\prime}(0)=1,\left(q_{\nu}^{\mu}\right)^{\prime}(0)=\frac{\Gamma\left(\frac{3}{2}+\nu\right) \Gamma\left(-\frac{1}{2}\right)}{\Gamma\left(\frac{1-\mu+\nu}{2}\right) \Gamma\left(\frac{1+\mu+\nu}{2}\right)},
\end{aligned}
$$

where $\nu=4 / \kappa$ and $\mu=1-\nu$. To see this, set $w(x)=w_{0}(x)\left(1+x^{2}\right)^{\frac{3}{2}+\frac{2}{\kappa}}$. Then $w_{0}$ is a solution to the inhomogeneous ODE:

$$
y^{\prime \prime}+\frac{2 x}{1+x^{2}} y^{\prime}+\frac{-\nu(\nu+1) x^{2}+1-3 \nu}{\left(1+x^{2}\right)^{2}} y=\frac{-\nu}{\left(1+x^{2}\right)^{\frac{5}{2}+\frac{2}{\kappa}}} .
$$

Thus, there are constants $C_{1}$ and $C_{2}$ such that

$$
\begin{aligned}
w_{0}(x) & =C_{1} p_{\nu}^{\mu}(x)+C_{2} q_{\nu}^{\mu}(x) \\
& -\int_{0}^{x} \frac{p_{\nu}^{\mu}(x) q_{\nu}^{\mu}(t)-q_{\nu}^{\mu}(x) p_{\nu}^{\mu}(t)}{W(t)} \frac{-\nu}{\left(1+t^{2}\right)^{\frac{5}{2}+\frac{2}{\kappa}}} d t,
\end{aligned}
$$

where the Wronskian $W$ is of the form $W(x)=W(0) \exp \left(-\int_{0}^{x} 2 t /\left(1+t^{2}\right)\right) d t=$ $W(0) /\left(1+x^{2}\right)$. After changing the limits,

$$
\begin{aligned}
w_{0}(x) & =c_{1} p_{\nu}^{\mu}(x)+c_{2} q_{\nu}^{\mu}(x) \\
& +\int_{x}^{\infty} \frac{p_{\nu}^{\mu}(x) q_{\nu}^{\mu}(t)-q_{\nu}^{\mu}(x) p_{\nu}^{\mu}(t)}{W(0)} \frac{-\nu}{\left(1+t^{2}\right)^{\frac{3}{2}+\frac{2}{\kappa}}} d t,
\end{aligned}
$$

where $W=W\left[p_{\nu}^{\mu}, q_{\nu}^{\mu}\right]$. We expand $p_{\nu}^{\mu}$ and $q_{\nu}^{\mu}$ as hypergeometric functions:

$$
p_{\nu}^{\mu}(x)=\left(1+x^{2}\right)^{\frac{1}{2} \nu}{ }_{2} F_{1}\left(\frac{\mu-\nu}{2},-\frac{\mu+\nu}{2} ; \frac{1}{2}-\nu ; \frac{1}{1+x^{2}}\right)
$$

and

$$
q_{\nu}^{\mu}(x)=\left(1+x^{2}\right)^{-\frac{1}{2} \nu-\frac{1}{2}}{ }_{2} F_{1}\left(\frac{1-\mu+\nu}{2}, \frac{1+\mu+\nu}{2} ; \frac{3}{2}+\nu ; \frac{1}{1+x^{2}}\right)
$$

on the set $\mathbb{R}_{+}$of all positive real numbers. Formally, the classical generalized Legendre function $P_{\nu}^{\mu}(z)$ of the first kind and $Q_{\nu}^{\mu}(z)$ of the second kind are linear combinations of $p_{\nu}^{\mu}(i z)$ and $q_{\nu}^{\mu}(i z)$. For special functions, see [11. In particular, the expansion (2.16) has an extension to all of $\mathbb{R}$ given by

$$
p_{\nu}^{\mu}(x)=x\left(1+x^{2}\right)^{\frac{\nu-1}{2}}=x\left(1+x^{2}\right)^{\frac{2}{\kappa}-\frac{1}{2}} .
$$

We will show $w(x)=\left(x^{2}+1\right) / 4$. One can easily get $c_{1}=0$ by the distortion theorem (1.5) and the unboundedness of $p_{\nu}^{\mu}(x)\left(1+x^{2}\right)^{\frac{1}{2}+\frac{2}{\kappa}}$ as $x \rightarrow \infty$. By the symmetry of SLE, we have $w^{\prime}(0)=0$. This information makes it possible to determine the value of $c_{2}$. Therefore, with the properties that $w$ is even and $w(x) /\left(1+x^{2}\right)$ is bounded, $w$ is the unique solution to the inhomogeneous ODE: $H w=1$. One can easily check that $w(x)=\left(x^{2}+1\right) / 4$ is the desired solution. 
Recall that the Schwarzian derivative, after we subtract a negligible term, is a complex martingale (1.20). Set

$$
w(x):=2\left(x^{2}+1\right)^{2} \mathbb{E} \int_{0}^{\infty} \frac{\left|f_{t}^{\prime}(x+i)\right|^{4}}{\left|f_{t}(x+i)+\sqrt{\kappa} B_{t}\right|^{6}} d t .
$$

As in (2.13), it follows from the scaling property of SLE that

$$
\mathbb{E} \int_{0}^{\infty} \frac{\left|f_{t}^{\prime}(z)\right|^{4}}{\left|f_{t}(z)+\sqrt{\kappa} B_{t}\right|^{6}} d t=\frac{1}{2 y^{4}} \frac{w(x / y)}{\left(1+x^{2} / y^{2}\right)^{2}} .
$$

As in the pre-Schwarzian, the function $w$ satisfies the inhomogeneous ODE: $H w=$ 1 , where $H$ is the second-order linear differential operator given by

$$
H v=-\frac{\kappa}{4}\left(1+x^{2}\right) v_{x x}+(2+2 \kappa) x v_{x}-\frac{(8+5 \kappa) x^{2}-(8+\kappa)}{1+x^{2}} v .
$$

To see this, let $v(x, s):=\mathbb{E}\left(\left|(x+i) f_{\tau_{s}}^{\prime}(x+i)\right|^{4} /\left|f_{\tau_{s}}(x+i)+\sqrt{\kappa} B_{\tau_{s}}\right|^{4}\right)$, where the stopping time $\tau_{s}$ is defined by (2.14). Then $v$ can be expressed as a Feynman-Kac type integral

$$
v(x, s)=\mathbb{E}\left(\exp \left[(8+\kappa) \int_{0}^{s} \frac{X_{u}^{2}-1}{X_{u}^{2}+1} d u-2 \sqrt{2 \kappa} \int_{0}^{s} \frac{X_{u}}{\sqrt{X_{u}^{2}+1}} d B_{u}\right]\right),
$$

where $X_{u}:=\operatorname{Re}\left(f_{\tau_{u}}(z)+\sqrt{\kappa} B_{\tau_{u}}\right) / \operatorname{Im}\left(f_{\tau_{u}}(z)+\sqrt{\kappa} B_{\tau_{u}}\right)$ is an Itô diffusion (2.15)). Therefore, $v$ satisfies the following parabolic equation with an initial condition:

$$
v_{s}=-H v \text { and } v(x, 0) \equiv 1 .
$$

On the other hand, with the properties that $w(x) /\left(1+x^{2}\right)^{2}$ is bounded and $w$ is even, $w$ is the unique solution to the inhomogeneous ODE: $H w=1$. It is easy to check that

$$
w(x)=\frac{8+3 \kappa}{32(6+\kappa)} x^{4}+\frac{16+3 \kappa}{16(6+\kappa)} x^{2}+\frac{24+3 \kappa}{32(6+\kappa)} .
$$

By (2.20) and (2.21), we obtain

$$
\mathbb{E} \int_{0}^{\infty} \frac{\left|f_{t}^{\prime}(z)\right|^{4}}{\left|f_{t}(z)+\sqrt{\kappa} B_{t}\right|^{6}} d t=\frac{1}{y^{4}} \frac{3 \kappa+8}{64(\kappa+6)}+\frac{1}{y^{2}\left(x^{2}+y^{2}\right)} \frac{1}{4(\kappa+6)} .
$$

For $x \neq 0$, we have

$$
\lim _{y \rightarrow 0} \mathbb{E} \int_{0}^{\infty} \frac{\left|y f_{t}^{\prime}(z)\right|^{4}}{\left|f_{t}(z)+\sqrt{\kappa} B_{t}\right|^{6}} d t=\frac{3 \kappa+8}{64(\kappa+6)},
$$

which proves the second part of Theorem 3 ,

Because of (2.22), it is useful to study the Itô derivatives

$$
d \frac{\left|f_{t}^{\prime}(z)\right|^{4}}{\left(f_{t}(z)-\overline{f_{t}(z)}\right)^{2}\left|f_{t}(z)+\sqrt{\kappa} B_{t}\right|^{2}} \quad \text { and } \quad d \frac{\left|f_{t}^{\prime}(z)\right|^{4}}{\left(f_{t}(z)-\overline{f_{t}(z)}\right)^{4}}
$$

in order to derive the Schwarzian expectation, and we now turn to this task. 


\section{Schwarzian expectation.}

Proof of Theorem [3, Use (1.9), (2.5), and Goluzin's identity (2.9) to derive

$$
\begin{aligned}
& d \frac{f_{t}^{\prime}(z) \overline{f_{t}^{\prime}(z)}}{\left(f_{t}(z)-\overline{f_{t}(z)}\right)^{2}} \frac{f_{t}^{\prime}(z) \overline{f_{t}^{\prime}(z)}}{\left(f_{t}(z)+\sqrt{\kappa} B_{t}\right)\left(\overline{f_{t}(z)}+\sqrt{\kappa} B_{t}\right)} \\
& =\frac{\left|f_{t}^{\prime}(z)\right|^{4}}{\left(f_{t}(z)-\overline{f_{t}(z)}\right)^{2}\left|f_{t}(z)+\sqrt{\kappa} B_{t}\right|^{2}} \\
& \times\left(\frac{\kappa+4}{\left(f_{t}(z)+\sqrt{\kappa} B_{t}\right)^{2}}+\frac{\kappa+4}{\left(\overline{f_{t}(z)}+\sqrt{\kappa} B_{t}\right)^{2}}+\frac{\kappa}{\left|f_{t}(z)+\sqrt{\kappa} B_{t}\right|^{2}}\right) d t \\
& +\frac{2 f_{t}^{\prime}(z)^{2} \overline{f_{t}^{\prime}(z)^{2}}}{\left(f_{t}(z)+\sqrt{\kappa} B_{t}\right)^{3}\left(\overline{f_{t}(z)}+\sqrt{\kappa} B_{t}\right)^{3}} d t+\text { martingale } \\
& =\frac{(\kappa+6) f_{t}^{\prime}(z)^{2} \overline{f_{t}^{\prime}(z)^{2}}}{\left(f_{t}(z)+\sqrt{\kappa} B_{t}\right)^{3}\left(\overline{f_{t}(z)}+\sqrt{\kappa} B_{t}\right)^{3}} d t \\
& +\frac{3 \kappa+8}{4} d \frac{f_{t}^{\prime}(z)^{2} \overline{f_{t}^{\prime}(z)^{2}}}{\left(f_{t}(z)-\overline{f_{t}(z)}\right)^{4}}+\text { martingale. }
\end{aligned}
$$

By the optional stopping theorem, the above equality implies that

$$
\begin{aligned}
\mathbb{E} \int_{0}^{T_{u}} & \frac{\left|f_{s}^{\prime}(z)\right|^{4}}{\left|f_{s}(z)+\sqrt{\kappa} B_{s}\right|^{6}} d s=\frac{3 \kappa+8}{64(\kappa+6)}\left(\frac{1}{y^{4}}-\mathbb{E} \frac{\left|f_{T_{u}}^{\prime}(z)\right|^{4}}{\left(\operatorname{Im} f_{T_{u}}(z)\right)^{4}}\right) \\
& +\frac{1}{4(\kappa+6)}\left(\frac{1}{y^{2}\left(x^{2}+y^{2}\right)}-\mathbb{E} \frac{\left|f_{T_{u}}^{\prime}(z)\right|^{4}}{\left(\operatorname{Im} f_{T_{u}}(z)\right)^{2}\left|f_{T_{u}}(z)+\sqrt{\kappa} B_{T_{u}}\right|^{2}}\right) .
\end{aligned}
$$

However, by (2.1), (1.20), and the optional stopping theorem, we obtain

$$
\begin{aligned}
\lim _{y \rightarrow 0} \mathbb{E} \mid y^{2} S f_{T_{u}}(z) & +\left.\frac{12}{3 \kappa+8}\left(\frac{y^{2}}{z^{2}}-\frac{y^{2} f_{T_{u}}^{\prime}(z)^{2}}{\left(f_{T_{u}}(z)+\sqrt{\kappa} B_{T_{u}}\right)^{2}}\right)\right|^{2} \\
& =\frac{576 \kappa}{(3 \kappa+8)^{2}} \lim _{y \rightarrow 0} \mathbb{E} \int_{0}^{T_{u}} \frac{\left|y f_{s}^{\prime}(z)\right|^{4}}{\left|f_{s}(z)+\sqrt{\kappa} B_{s}\right|^{6}} d s \\
& =\frac{9 \kappa}{(\kappa+6)(3 \kappa+8)},
\end{aligned}
$$

unless $x=0$. One can easily check that $\mathbb{E}\left|y f_{T_{u}}^{\prime}(z) /\left(f_{T_{u}}(z)+\sqrt{\kappa} B_{T_{u}}\right)\right|^{4} \rightarrow 0$ as $y \rightarrow 0$. (See the calculation used in (2.10).) This completes the proof of the first part, equation (1.18). The second part, equation (1.19), was proved in (2.22).

\section{Schwarzian and $\mathcal{B M O}$.}

Proof of Theorem 4. Suppose a stopping time $\tau$ is given. By the strong Markov property, we have

$$
\begin{aligned}
y^{4} \int_{\tau}^{\infty} & \mathbb{E}\left[\frac{\left|f_{t}^{\prime}(z)\right|^{4}}{\left|f_{t}(z)+\sqrt{\kappa} B_{t}\right|^{6}} \mid \mathcal{F}_{\tau}\right] d t \\
& =y^{4}\left|f_{\tau}^{\prime}(z)\right|^{4} \int_{0}^{\infty} \mathbb{E} \frac{\left|f_{t}^{\prime}\left(f_{\tau}(z)+\sqrt{\kappa} B_{\tau}\right)\right|^{4}}{\left|f_{t}\left(f_{\tau}(z)+\sqrt{\kappa} B_{\tau}\right)+\sqrt{\kappa} B_{t}\right|^{6}} d t .
\end{aligned}
$$

The Schwarz lemma implies $y\left|f_{\tau}^{\prime}(z)\right| \leq \operatorname{Im}\left(f_{\tau}(z)+\sqrt{\kappa} B_{\tau}\right)$. Therefore, it suffices to show that

$$
\mathbb{E} \int_{0}^{\infty} \frac{\left|y f_{t}^{\prime}(z)\right|^{4}}{\left|f_{t}(z)+\sqrt{\kappa} B_{t}\right|^{6}} d t
$$


is uniformly bounded above by a universal constant $C=C(z)$. By (2.22), we have

$$
\mathbb{E}\left(\left|S_{\infty}-S_{\tau}\right|^{2} \mid \mathcal{F}_{\tau}\right) \leq \frac{9 \kappa}{(3 \kappa+8)(\kappa+6)}+\frac{y^{2}}{x^{2}+y^{2}} \frac{144 \kappa}{(\kappa+6)(3 \kappa+8)^{2}},
$$

and $S$ is a $\mathcal{B M O}$ martingale.

\section{EXPONENTIAL DECAY OF CORRELATIONS}

In many problems, random variables with exponential decay of correlations are the appropriate substitutes for the independent random variables. For example, B. Schmuland and W. Sun proved the law of the iterated logarithm for a random field with exponential decay of correlations. See 32 .

Proof of Theorem 5. By (1.17), (1.9), and the chordal version of Goluzin's identity (2.9), we obtain

$$
\begin{aligned}
\mathbb{E}\left(L_{t}\left(z_{1}\right) \overline{L_{t}\left(z_{2}\right)}\right) & =\frac{4}{(\sqrt{\kappa / 4}+\sqrt{4 / \kappa})^{2}} \mathbb{E} \int_{0}^{t} \frac{y_{1} y_{2} f_{s}^{\prime}\left(z_{1}\right) \overline{f_{s}^{\prime}\left(z_{2}\right)}}{\left(f_{s}\left(z_{1}\right)+\sqrt{\kappa} B_{s}\right)^{2}\left(\overline{f_{s}\left(z_{2}\right)}+\sqrt{\kappa} B_{s}\right)^{2}} d s \\
& =\frac{2}{(\sqrt{\kappa / 4}+\sqrt{4 / \kappa})^{2}} \mathbb{E}\left[\frac{y_{1} y_{2} f_{t}^{\prime}\left(z_{1}\right) \overline{f_{t}^{\prime}\left(z_{2}\right)}}{\left(f_{t}\left(z_{1}\right)-\overline{f_{t}\left(z_{2}\right)}\right)^{2}}-\frac{y_{1} y_{2}}{\left(z_{1}-\overline{z_{2}}\right)^{2}}\right] .
\end{aligned}
$$

Recall the definition of the hyperbolic distance (1.21) or

$$
\frac{y_{1} y_{2}}{\left|z_{1}-\overline{z_{2}}\right|^{2}}=\frac{1}{4} \cosh ^{-2}\left(\frac{d_{\mathbb{H}}\left(z_{1}, z_{2}\right)}{2}\right) \text {. }
$$

On the other hand,

$$
\limsup _{t \rightarrow \infty}\left|\mathbb{E}\left(\frac{y_{1} y_{2} f_{t}^{\prime}\left(z_{1}\right) \overline{f_{t}^{\prime}\left(z_{2}\right)}}{\left(f_{t}\left(z_{1}\right)-\overline{f_{t}\left(z_{2}\right)}\right)^{2}}\right)\right| \leq \frac{1}{4} \limsup _{t \rightarrow \infty} \mathbb{E}\left(\left|\frac{y_{1} f_{t}^{\prime}\left(z_{1}\right)}{\operatorname{Im} f_{t}\left(z_{1}\right)}\right|\left|\frac{y_{2} f_{t}^{\prime}\left(z_{2}\right)}{\operatorname{Im} f_{t}\left(z_{2}\right)}\right|\right)=0,
$$

which implies (1.22). It follows from identity (2.7) that $\left|y f_{t}^{\prime}(z) /\left(\operatorname{Im} f_{t}(z)\right)\right|$ is monotone decreasing in $t$. Hence,

$$
\left|\mathbb{E}\left(\frac{y_{1} y_{2} f_{T_{0}^{*}}^{\prime}\left(z_{1}\right) \overline{f_{T_{0}^{*}}^{\prime}\left(z_{2}\right)}}{\left(f_{T_{0}^{*}}\left(z_{1}\right)-\overline{f_{T_{0}^{*}}\left(z_{2}\right)}\right)^{2}}\right)\right| \leq \frac{1}{4} \prod_{j=1}^{2} \sqrt{\mathbb{E}\left(\left|\frac{y_{j} f_{T_{0}\left(z_{j}\right)}^{\prime}\left(z_{j}\right)}{\operatorname{Im} f_{T_{0}\left(z_{j}\right)}\left(z_{j}\right)}\right|^{2}\right)} .
$$

In the proof of Theorem 1 we have shown that $\mathbb{E}\left|y f_{T_{0}(z)}^{\prime}(z)\right|^{2}$ converges to 0 as $y \rightarrow 0$. Estimate (1.23) now follows by scale invariance.

Proof of Theorem 6. By (1.20) and (1.9), we obtain

$$
\mathbb{E}\left(S f_{t}\left(z_{1}\right) \overline{S f_{t}\left(z_{2}\right)}\right)=\frac{576 \kappa}{(3 \kappa+8)^{2}} \mathbb{E} \int_{0}^{t} \frac{y_{1}^{2} y_{2}^{2} f_{s}^{\prime}\left(z_{1}\right)^{2} \overline{f_{s}^{\prime}\left(z_{2}\right)^{2}}}{\left(f_{s}\left(z_{1}\right)+\sqrt{\kappa} B_{s}\right)^{3}\left(\overline{f_{s}\left(z_{2}\right)}+\sqrt{\kappa} B_{s}\right)^{3}} d s .
$$

Use Goluzin's identity to derive

$$
\begin{aligned}
d \frac{f_{t}^{\prime}\left(z_{1}\right) \overline{f_{t}^{\prime}\left(z_{2}\right)}}{\left(f_{t}\left(z_{1}\right)-\overline{f_{t}\left(z_{2}\right)}\right)^{2}} & \frac{f_{t}^{\prime}\left(z_{1}\right) \overline{f_{t}^{\prime}\left(z_{2}\right)}}{\left(f_{t}\left(z_{1}\right)+\sqrt{\kappa} B_{t}\right)\left(\overline{f_{t}\left(z_{2}\right)}+\sqrt{\kappa} B_{t}\right)} \\
& =(\kappa+6) \frac{f_{t}^{\prime}\left(z_{1}\right)^{2}}{f_{t}^{\prime}\left(z_{2}\right)^{2}} \\
& +\frac{3 \kappa+8}{4} d \frac{f_{t}^{\prime}\left(z_{1}\right)^{2} \overline{f_{t}^{\prime}\left(z_{2}\right)^{2}}}{\left(f_{t}\left(z_{1}\right)-\overline{f_{t}\left(z_{2}\right)}\right)^{4}}+\text { martingale. }
\end{aligned}
$$


Taking the expectation in the above equation, we obtain

$$
\begin{aligned}
\mathbb{E} \int_{0}^{t} & \frac{y_{1}^{2} y_{2}^{2} f_{s}^{\prime}\left(z_{1}\right)^{2} \overline{f_{s}^{\prime}\left(z_{2}\right)^{2}}}{\left(f_{s}\left(z_{1}\right)+\sqrt{\kappa} B_{s}\right)^{3}\left(\overline{f_{s}\left(z_{2}\right)}+\sqrt{\kappa} B_{s}\right)^{3}} d s \\
& =\frac{3 \kappa+8}{4(\kappa+6)} \frac{y_{1}^{2} y_{2}^{2}}{\left(z_{1}-\overline{z_{2}}\right)^{4}}-\frac{1}{\kappa+6} \frac{y_{1}^{2} y_{2}^{2}}{\left(z_{1}-\overline{z_{2}}\right)^{2} z_{1} \overline{z_{2}}} \\
& -\frac{3 \kappa+8}{4(\kappa+6)} \mathbb{E} \frac{y_{1}^{2} y_{2}^{2} f_{t}^{\prime}\left(z_{1}\right)^{2} \overline{f_{t}^{\prime}\left(z_{2}\right)^{2}}}{\left(f_{t}\left(z_{1}\right)-\overline{f_{t}\left(z_{2}\right)}\right)^{4}} \\
& +\frac{1}{\kappa+6} \mathbb{E} \frac{y_{1} y_{2} f_{t}^{\prime}\left(z_{1}\right) \overline{f_{t}^{\prime}\left(z_{2}\right)}}{\left(f_{t}\left(z_{1}\right)-\overline{f_{t}\left(z_{2}\right)}\right)^{2}} \frac{y_{1} y_{2} f_{t}^{\prime}\left(z_{1}\right) \overline{f_{t}^{\prime}\left(z_{2}\right)}}{\left(f_{t}\left(z_{1}\right)+\sqrt{\kappa} B_{t}\right)\left(\overline{f_{t}\left(z_{2}\right)}+\sqrt{\kappa} B_{t}\right)} .
\end{aligned}
$$

The estimates (1.24) and (1.25) can be easily obtained by the same method used in the proof of Theorem 5 .

\section{HÖLDER CONTINUITY}

S. Rohde and O. Schramm's estimate. The derivative expectation $\mathbb{E}\left[\left|g_{t}^{\prime}(1)\right|^{p}\right]$ was computed by G. F. Lawler, O. Schramm, and W. Werner to obtain the crossing exponent for $\mathrm{SLE}_{\kappa}$, which is closely related to the Brownian intersection exponent. This computation led them to prove the Mandelbrot conjecture. To obtain more information about the regularity of the backward flows, the derivative expectation or moment generating function for $\left|g_{t}^{\prime}(z)\right|$ for an arbitrary complex number $z$ has been computed in 31]. Using this, S. Rohde and O. Schramm proved that $g_{t}^{-1}$ is almost surely Hölder continuous when $\kappa \neq 4$.

For $z \in \mathbb{H}$ and $u \in \mathbb{R}$, set

$$
T_{u}=T_{u}(z):=\sup \left\{t \in \mathbb{R}: \operatorname{Im}\left(g_{t}(z)\right) \geq e^{u}\right\}
$$

and

$$
X_{u}=\operatorname{Re}\left(g_{T_{u}(z)}(z)-\sqrt{\kappa} B_{T_{u}}\right) .
$$

The stopping time $T_{u}$ is well-defined since $\operatorname{Im} g_{t}(z)$ is monotone decreasing in $t$ for each $z \in \mathbb{H}$. It is well known that for all $z \in \mathbb{H}$, almost surely $T_{u} \neq \pm \infty$.

Theorem (S. Rohde, O. Schramm). Suppose $z=x+i y \in \mathbb{H}$ with $y<1$ given. For each $b \in \mathbb{R}$, define $p$ and $q$ by

$$
p:=2 b+\kappa b(1-b) / 2, \quad q:=4 b+\kappa b(1-2 b) / 2 .
$$

Then

$$
y^{p} \mathbb{E}\left[\left(1+X_{0}^{2}\right)^{b}\left|g_{T_{0}(z)}^{\prime}(z)\right|^{p}\right]=\left(1+x^{2} / y^{2}\right)^{b} y^{q} .
$$

Corollary (S. Rohde, O. Schramm). For $b \in[0,1+4 / \kappa]$, there is a constant $C(\kappa, b)$, depending only on $\kappa$ and $b$, such that the following derivative upper bound estimate holds for all $t \in[0,1], y, \delta \in(0,1]$ and $x \in \mathbb{R}$.

$$
\mathbb{P}\left[\left|f_{t}^{\prime}(x+i y)\right| \geq \delta y^{-1}\right] \leq C(\kappa, b)\left(1+x^{2} / y^{2}\right)^{b}(y / \delta)^{q} \theta(\delta, p-q),
$$

where

$$
\theta(\delta, s)= \begin{cases}\delta^{-s} & s>0 \\ 1+|\log \delta| & s=0 \\ 1 & s<0\end{cases}
$$


Theorem (S. Rohde, O. Schramm). For every $\kappa \neq 4$, there exists $\widetilde{h}(\kappa)>0$ such that for each bounded set $D \subset \mathbb{H}$ and each $t>0$, almost surely $f_{t}$ is Hölder continuous with Hölder exponent $\widetilde{h}(\kappa)$ on $D$,

$$
\left|f_{t}(z)-f_{t}(w)\right| \leq C|z-w|^{\widetilde{h}(\kappa)}, \quad z, w \in D
$$

where $C$ is a random constant depending on $t$ and D. Moreover,

$$
\lim _{\kappa \searrow 0} \widetilde{h}(\kappa)=\frac{1}{2} \text { and } \lim _{\kappa \nearrow \infty} \widetilde{h}(\kappa)=1 .
$$

Here, by bounded set we mean bounded in the sense of the Euclidean metric on $\mathbb{R}^{2}$ (not the hyperbolic metric on $\mathbb{H}$ ). When $\kappa=0$, the Schramm-Loewner evolution is not a stochastic process anymore. In this case, the backward flow $f_{t}$ coincides with $g_{t}^{-1}$ and is given by

$$
f_{t}(z)=\sqrt{z^{2}-4 t}
$$

which has a Hölder exponent of $1 / 2$. However, it has a local Hölder exponent of 1 except at $z= \pm 2 \sqrt{t}$. These two points are mapped into the base point, where the geometry of Loewner evolution is different from any other points. The geometry of the base point will not be taken into consideration with the conditioning on the event that $\operatorname{Im} f_{1}(z) \geq c$ on $D$. For example, to compute the size of the hull boundary, S. Rohde and $\mathrm{O}$. Schramm considered the collection $W_{c}$ of the Whitney squares $Q$ of $H_{1}$ such that $\operatorname{dist}\left(Q, K_{1}\right) \leq 1$ and $\sup \{\operatorname{Im} z: z \in Q\} \geq c$. For $\delta>0$, they introduced

$$
S_{c}(\delta):=\sum_{Q \in W_{c}} d(Q)^{\delta}
$$

and computed the convergence exponent $\delta(\kappa)$ such that $\mathbb{E}\left[S_{c}(\delta)\right]=\infty$ if and only if $\delta \leq \delta(\kappa)$. It is conjectured that the convergent exponent $\delta(\kappa)$ is the Hausdorff dimension for the boundary of the hull.

From (4.3), we have the following estimate for the derivative expectation: For each bounded set $D \subset \mathbb{H}$,

$$
\mathbb{E}\left|g_{T_{0}}^{\prime}(z)\right|^{p} \leq C y^{p\left(1-\frac{1-\sqrt{1-2 p / \mu}}{p / \mu}\right)},
$$

where $\mu=\kappa / 4+2+4 / \kappa$ and $C$ is a constant depending on $D$. Without rigorous proof, we will derive the estimate on the distributions for the derivatives from (4.4) in the next subsection. With the conditioning on the event $\operatorname{Im} f_{1}(z) \geq c$ on $D$, we will rigorously derive the conjectured sharp estimate on the Hölder exponent in the last subsection. The next subsection may be of help to formulate Theorem 7 However, the reader may wish to skip it.

Distributions for derivatives. Set the distribution $G(\lambda)=\mathbb{P}\left[\left|g_{T_{0}}^{\prime}(z) / y\right|>\lambda\right]$. Then estimate (4.4) says

$$
\int_{0}^{\infty} p G(\lambda) \lambda^{p-1} d \lambda \leq C y^{-\mu+\mu \sqrt{1-2 p / \mu}}
$$

Use the substitution $r=1-2 p / \mu(0<r<1)$ and $\nu=\lambda^{-\mu / 2}$ to obtain

$$
\int_{0}^{\infty} y^{\mu} \frac{G(\lambda)}{\nu} \nu^{r-1} d \nu \leq C \frac{e^{-\sqrt{a r}}}{1-r}
$$

where $\sqrt{a}=\mu \log y^{-1}$. We will estimate $y^{\mu} G(\lambda) / \nu$ by taking the inverse Mellin transform of $e^{-\sqrt{a r}} /(1-r)$ formally on both sides of the above inequality. 
Let us recall the definition of the Mellin transform and the Laplace transform. The Mellin transform $M[f]$ is defined as

$$
M[f](z)=\int_{0}^{\infty} t^{z-1} f(t) d t
$$

and its inverse transform $M^{-1}[\phi]$ is defined as

$$
M^{-1}[\phi](t)=\frac{1}{2 \pi i} \int_{c-i \infty}^{c+i \infty} t^{-z} \phi(z) d z .
$$

The transform $\phi(z)=M[f](z)$ exists if the integral

$$
\int_{0}^{\infty}|f(x)| x^{k-1} d x
$$

is bounded for some $k>0$, in which case the inverse $f(t)=M^{-1}[\phi](t)$ exists when $c>k$. The Laplace transform $\mathcal{L}$ is defined by

$$
\mathcal{L}[f](s)=\int_{0}^{\infty} f(t) e^{-s t} d t .
$$

If $f$ is a piecewise continuous function on every finite interval in $[0, \infty)$ satisfying $|f(t)| \leq C e^{a t}$ for all $t \in[0, \infty)$, then $\mathcal{L}[f](s)$ exists for all $s>a$. The Laplace transform is unique, and its inverse transform $\mathcal{L}^{-1}$ is defined as

$$
\mathcal{L}^{-1}[\phi](t)=\frac{1}{2 \pi i} \int_{c-i \infty}^{c+i \infty} e^{t z} \phi(z) d z .
$$

The error function and the complementary error function are defined as

$$
\operatorname{erf}(x)=\frac{2}{\sqrt{\pi}} \int_{0}^{x} e^{-t^{2}} d t, \quad \operatorname{erfc}(x)=1-\operatorname{erf}(x)
$$

We will use the following elementary properties of the complementary error function.

(1) It satisfies the identity: $\operatorname{erfc}(-x)=2-\operatorname{erfc}(x)$.

(2) It has the tail estimate: For $x>0$,

$$
\frac{2}{\sqrt{\pi}} \frac{e^{-x^{2}}}{x+\sqrt{x^{2}+2}}<\operatorname{erfc}(x)<\frac{2}{\sqrt{\pi}} \frac{e^{-x^{2}}}{x+\sqrt{x^{2}+\frac{4}{\pi}}} .
$$

To compute the inverse Mellin transform of $e^{-\sqrt{a r}} /(1-r)$, we need the following lemma.

Lemma 4.1. The inverse Laplace transform of $e^{-\sqrt{a r}} /(1-r)$ on $0<r<1$ is

$$
\frac{1}{2 \pi i} \int_{r-i \infty}^{r+i \infty} \frac{e^{x z-\sqrt{a z}}}{1-z} d z=\frac{1}{2} e^{x}\left[e^{-\sqrt{a}} \operatorname{erfc}\left(\sqrt{x}-\frac{1}{2} \sqrt{\frac{a}{x}}\right)-e^{\sqrt{a}} \operatorname{erfc}\left(\frac{1}{2} \sqrt{\frac{a}{x}}+\sqrt{x}\right)\right],
$$

where erfc is the complementary error function.

Proof. It is well known that

$$
\mathcal{L}^{-1}\left(\frac{e^{-\sqrt{a p}}}{p}\right)(x)=\operatorname{erfc}\left(\frac{1}{2} \sqrt{\frac{a}{x}}\right) .
$$


See p. 264 in [12. It follows from the basic properties of the Laplace transform that

$$
\begin{aligned}
\mathcal{L}^{-1}\left(\frac{e^{-\sqrt{a p}}}{p-1}\right)(x) & =e^{x} \int_{0}^{x} e^{-t} \mathcal{L}^{-1}\left(e^{-\sqrt{a p}}\right)(t) d t \\
& =e^{x} \int_{0}^{x} e^{-t} \frac{d}{d t} \mathcal{L}^{-1}\left(\frac{e^{-\sqrt{a p}}}{p}\right)(t) d t \\
& =e^{x} \int_{0}^{x} e^{-t} \frac{d}{d t} \operatorname{erfc}\left(\frac{1}{2} \sqrt{\frac{a}{t}}\right) d t \\
& =\frac{1}{2} e^{x}\left[e^{-\sqrt{a}} \operatorname{erfc}\left(\frac{1}{2} \sqrt{\frac{a}{x}}-\sqrt{x}\right)+e^{\sqrt{a}} \operatorname{erfc}\left(\frac{1}{2} \sqrt{\frac{a}{x}}+\sqrt{x}\right)\right] .
\end{aligned}
$$

Suppose $p>1$ and $0<r<1$. Then, using a contour integral, we obtain

$$
\begin{aligned}
\frac{1}{2 \pi i} \int_{r-i \infty}^{r+i \infty} \frac{e^{x z-\sqrt{a z}}}{1-z} d z=\operatorname{Res}_{z=1}\left(\frac{e^{x z-\sqrt{a z}}}{z-1}\right)-\frac{1}{2 \pi i} \int_{p-i \infty}^{p+i \infty} \frac{e^{x z-\sqrt{a z}}}{z-1} d z \\
=e^{x-\sqrt{a}}-\frac{1}{2} e^{x}\left[e^{-\sqrt{a}} \operatorname{erfc}\left(\frac{1}{2} \sqrt{\frac{a}{x}}-\sqrt{x}\right)+e^{\sqrt{a}} \operatorname{erfc}\left(\frac{1}{2} \sqrt{\frac{a}{x}}+\sqrt{x}\right)\right] \\
\quad=\frac{1}{2} e^{x} e^{-\sqrt{a}}\left[2-\operatorname{erfc}\left(\frac{1}{2} \sqrt{\frac{a}{x}}-\sqrt{x}\right)\right]-\frac{1}{2} e^{x} e^{\sqrt{a}} \operatorname{erfc}\left(\frac{1}{2} \sqrt{\frac{a}{x}}+\sqrt{x}\right) \\
=\frac{1}{2} e^{x} e^{-\sqrt{a}} \operatorname{erfc}\left(\sqrt{x}-\frac{1}{2} \sqrt{\frac{a}{x}}\right)-\frac{1}{2} e^{x} e^{\sqrt{a}} \operatorname{erfc}\left(\frac{1}{2} \sqrt{\frac{a}{x}}+\sqrt{x}\right) .
\end{aligned}
$$

This completes the proof.

Fix $\kappa \neq 4$. By scaling, we may assume $D=[-1,1] \times(0,1]$. With $\nu=\lambda^{-\mu / 2}$, $\sqrt{a}=\mu \log y^{-1}$, and $e^{-x}=\nu$, we take the inverse Mellin transform on both sides of (4.5) to formally derive the following estimate without rigorous proof:

$$
\mathbb{P}\left[\left|\frac{g_{T_{0}}^{\prime}(z)}{y}\right|>\lambda\right] \leq C\left(\operatorname{erfc}\left(\sqrt{\frac{\mu}{2}} \frac{\log (\lambda y)}{\sqrt{\log \lambda}}\right)-\frac{1}{y^{2 \mu}} \operatorname{erfc}\left(\sqrt{\frac{\mu}{2}} \frac{\log (\lambda / y)}{\sqrt{\log \lambda}}\right)\right) .
$$

From the above estimate, it is not hard to obtain the following estimate. The reader can find the details in the next subsection. Let $c>0$. Then

$$
\begin{aligned}
& \mathbb{P}\left[\left|\frac{f_{1}^{\prime}(z)}{y}\right|>\lambda \mid\right.\left.\inf _{z \in D} \operatorname{Im} f_{1}(z) \geq c\right] \\
& \leq C\left(\operatorname{erfc}\left(\sqrt{\frac{\mu}{2}} \frac{\log (\lambda y)}{\sqrt{\log \lambda}}\right)-\frac{1}{y^{2 \mu}} \operatorname{erfc}\left(\sqrt{\frac{\mu}{2}} \frac{\log (\lambda / y)}{\sqrt{\log \lambda}}\right)\right) .
\end{aligned}
$$

With $\lambda=y^{h-2}$ and $y=2^{-n}$, the above estimate gives

$$
\mathbb{P}\left[\left|\frac{f_{1}^{\prime}(z)}{y}\right|>\lambda \mid \inf _{z \in D} \operatorname{Im} f_{1}(z) \geq c\right] \leq C 2^{-n \frac{\mu}{2} \frac{(1-h)^{2}}{2-h}} .
$$

Consider the Whitney decomposition $\left\{Q_{j, n}\right\}\left(n \geq 0,1 \leq j \leq 2^{n}\right)$ of $D$. Denote the center of the Whitney square $Q_{j, n}$ by $z_{j, n}$. By the Borel-Cantelli lemma and the distortion theorem, $f_{1}$ is almost surely $h$-Hölder in $D$ on the event that $\operatorname{Im} f_{1}(z) \geq c$ in $D$, if

$$
\sum_{n=0}^{\infty} \sum_{j=1}^{2^{n}} \mathbb{P}\left[\left|f_{1}^{\prime}\left(z_{j, n}\right)\right|>2^{n(1-h)} \mid \inf _{z \in D} \operatorname{Im} f_{1}(z) \geq c\right]<\infty
$$



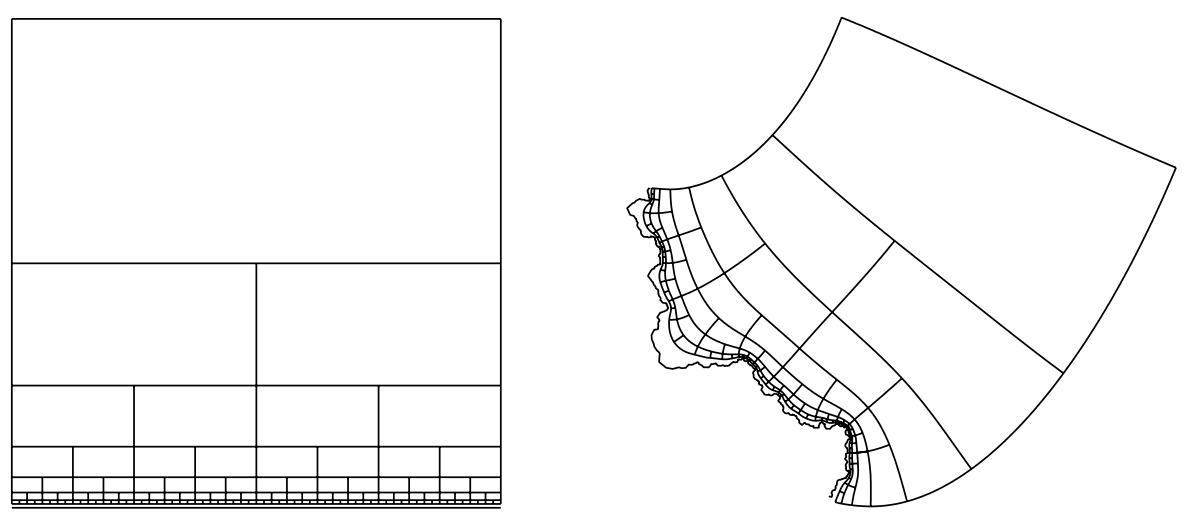

FiguRE 3. Whitney decomposition of $\mathbb{H}$ and its image under SLE.

This is summable if

$$
1-\frac{\mu}{2} \frac{(1-h)^{2}}{2-h}<0
$$

or

$$
h<h(\kappa)=1-\frac{1}{\mu}-\sqrt{\frac{1}{\mu^{2}}+\frac{2}{\mu}},
$$

where $\mu=\kappa / 4+2+4 / \kappa$. This motivates the formulation of Theorem 7

Remark 4.2. Note that $\lim _{\kappa \rightarrow 0} h(\kappa)=1, \lim _{\kappa \rightarrow \infty} h(\kappa)=1$, and $\lim _{\kappa \rightarrow 4} h(\kappa)=0$. Also, the exponent $h(\kappa)$ satisfies the Duplantier duality. I. Binder and B. Duplantier derived the same formula from the multifractal spectrum of $\mathrm{SLE}_{\kappa}$ independently. Indeed, the exponent $h(\kappa)$ satisfies $f(1 / h(\kappa))=0$ for the multifractal spectrum $f$ of $\mathrm{SLE}_{\kappa}$. Here, $f(\alpha)=f_{\text {mixed }}(\alpha, 0)$ for the mixed multifractal spectrum $f_{\text {mixed }}$ in (1.1). Hence, $f(\alpha)=\alpha+b-b \alpha^{2} /(2 \alpha-1)$, where $b=(25-c) / 12$ and $c=$ $1-6(\sqrt{\kappa / 4}-\sqrt{4 / \kappa})^{2}$. However, their result is not rigorous. We note that J. Lind has also obtained Theorem 7 independently. She has shown that $\left(f_{t}(\sqrt{z})+\sqrt{\kappa} B_{t}\right)^{2}$ is almost surely $h$-Hölder continuous provided $h<h(\kappa)$. See [28] for this.

Conjectured sharp estimate for the Hölder exponent. We will prove Theorem 7 rigorously in this subsection.

Proof of Theorem 7. Fix $\kappa \neq 4$. By scaling, we may assume $D=[-1 / 4,1 / 4] \times(0,1]$. Consider the Whitney decomposition $\left\{Q_{j, n}\right\}$ of $D$ such that $Q_{j, n}$ is a square of side length $2^{-n-1}\left(n \geq 0,1 \leq j \leq 2^{n}\right)$. We denote the center of the Whitney square $Q_{j, n}$ by $z_{j, n}$. Recall that $f_{1}$ is almost surely $h$-Hölder in $D$ on the event that $\operatorname{Im} f_{1}(z) \geq c$ in $D$ provided

$$
\sum_{n=0}^{\infty} \sum_{j=1}^{2^{n}} \mathbb{P}\left[\left|f_{1}^{\prime}\left(z_{j, n}\right)\right|>2^{n(1-h)} \mid \inf _{z \in D} \operatorname{Im} f_{1}(z) \geq c\right]<\infty .
$$

Take $m \in \mathbb{Z}$ such that $e^{m} \leq c<e^{m+1}$. Recall the stopping time (4.1) and the fact that $f_{1}^{\prime}$ has the same distribution as $g_{-1}^{\prime}$. As in the proof of Corollary 3.5 in [31, we may assume $T_{0}\left(z_{j, n}\right) \geq 1$ with probability one. Let $E$ be the event 
$E:=\left\{\inf _{z \in D} \operatorname{Im} f_{1}(z) \geq c\right\}$. For fixed $z=z_{j, n}$ and $k=m, \cdots, 0$, let $E_{k}$ be the event $E_{k}:=\left\{T_{k}(z) \leq 1<T_{k+1}(z)\right\}$. Then

$$
\begin{aligned}
& \mathbb{P}\left[y\left|f_{1}^{\prime}(z)\right|>\lambda \mid E\right] \\
& \quad \leq C_{1} \sum_{k=m}^{0} \mathbb{P}\left[y\left|g_{T_{k}(z)}^{\prime}(z)\right|>C_{2} \lambda \mid E E_{k}\right] \mathbb{P}\left[E_{k} \mid E\right] \\
& \quad \leq C \sum_{k=m}^{0} \lambda^{-p} e^{k p} F_{p}\left(e^{-k} z\right),
\end{aligned}
$$

where the moment generating function $F_{p}$ is given by $F_{p}(z)=y^{p} \mathbb{E}\left[\left|g_{T_{0}(z)}^{\prime}(z)\right|^{p}\right]$.

It follows from the above estimate and the estimate (4.4) that

$$
\sum_{n=0}^{\infty} \sum_{j=1}^{2^{n}} \mathbb{P}\left[\left|f_{1}^{\prime}\left(z_{j, n}\right)\right|>2^{n(1-h)} \mid E\right] \leq \sum_{n=0}^{\infty} C 2^{n(1+p h-2 p+\mu-\mu \sqrt{1-2 p / \mu})} .
$$

The right-hand side in the above inequality is summable if

$$
h<h_{\kappa}(p):=2-\frac{1}{p}+\frac{\mu}{p}\left(\sqrt{1-2 \frac{p}{\mu}}-1\right) .
$$

As a function of $p, h_{\kappa}(p)$ has the derivative

$$
h_{\kappa}^{\prime}(p)=\left(1-\frac{p}{\sqrt{1-2 \frac{p}{\mu}}}-\mu\left(\sqrt{1-2 \frac{p}{\mu}}-1\right)\right) \frac{1}{p^{2}}
$$

and takes the critical value at $p=p_{c}$, where

$$
p_{c}=-\left(2+\frac{1}{\mu}\right)+(\mu+1) \sqrt{\frac{1}{\mu^{2}}+\frac{2}{\mu}} .
$$

Furthermore, the critical value is

$$
\begin{aligned}
h_{\kappa}\left(p_{c}\right) & =2-\frac{1}{p_{c}}+\frac{1}{p_{c}}\left(1-\frac{p_{c}}{\sqrt{1-2 \frac{p_{c}}{\mu}}}\right) \\
& =2-\frac{1}{\sqrt{1-2 \frac{p_{c}}{\mu}}}=1-\frac{1}{\mu}-\sqrt{\frac{1}{\mu^{2}}+\frac{2}{\mu}}=h(\kappa),
\end{aligned}
$$

which gives the conjectured sharp estimate for the Hölder exponent.

Remark 4.3. For the backward flow of $\mathrm{SLE}_{4}$ one expects a continuity property of logarithmic type instead of Hölder continuity.

\section{ACKNOWLEDGEMENT}

I would like to thank my former advisor Peter W. Jones, who provided continuous wisdom throughout my project. I am grateful to Richard Beals, Ilia Binder, and Paul Müller for insightful conversations. I am especially thankful to Håkan Hedenmalm, who provided a useful suggestion that led me to extend my research to a greater scope. I also would like to thank the referees for their very helpful comments on the manuscript. 


\section{REFERENCES}

[1] V. Beffara, The dimension of the SLE curves, preprint, math.PR/0211322 (2002).

[2] I. Binder, Rotational spectrum of planar domains, Ph.D. thesis, Caltech, 1997.

[3] C. J. Bishop and P. W. Jones, Harmonic measure, $L^{2}$ estimates and the Schwarzian derivative, J. Anal. Math. 62 (1994), 77-113. MR1269200 (95f:30034)

[4] W Wiggly sets and limit sets, Ark. Mat. 35 (1997), no. 2, 201-224. MR.1478778 (99f:30066)

[5] C. J. Bishop, P. W. Jones, R. Pemantle, and Y. Peres, The dimension of the Brownian frontier is greater than 1, J. Funct. Anal. 143 (1997), no. 2, 309-336. MR.1428819 (97k:60105)

[6] L. de Branges, A proof of the Bieberbach conjecture, Acta Math. 154 (1985), no. 1, 137-152. MR7772434 (86h:30026)

[7] B. Duplantier, Conformally invariant fractals and potential theory, Phys. Rev. Lett. 84 (2000), no. 7, 1363-1367. MR.1740371 (2001c:82040)

[8] _ Conformal fractal geometry and boundary quantum gravity, Fractal geometry and applications: a jubilee of Benoît Mandelbrot, Part 2: Multifractals, probability and statistical mechanics, applications (Providence), Proc. Sympos. Pure Math., vol. 72, Amer. Math. Soc., 2004, pp. 365-482. MR2112128 (2005m:82057)

[9] B. Duplantier and I. Binder, Harmonic measure and winding of conformally invariant curves, Phys. Rev. Lett. 89 (2002), 264101.

[10] R. Durrett, Brownian motion and martingales in analysis, Wadsworth, Belmont, 1984. MR.750829 (87a:60054)

[11] A. Erdélyi, W. Magnus, F. Oberhettinger, and F. G. Tricomi, Higher transcendental functions. Vol. I., McGraw-Hill Book Company, Inc., New York-Toronto-London, 1953, based in part on notes left by Harry Bateman. MR698779 (84h:33001a)

[12] G. B. Folland, Fourier analysis and its applications, Wadsworth \& Brooks/Cole Advanced Books \& Software, Pacific Grove, 1992. MR1145236 (93f:42001)

[13] J. Garnett and D. E. Marshall, Harmonic measure, Cambridge University Press, Cambridge, 2005. MR2150803 (2006g:31002)

[14] G. M. Goluzin, Geometric theory of functions of a complex variable, Translations of Mathematical Monographs, vol. 26, Amer. Math. Soc., Providence, 1969. MR0247039 (40:308)

[15] J. Graczyk and P. W. Jones, Dimension of the boundary of quasiconformal Siegel disks, Invent. Math. 148 (2002), no. 3, 465-493. MR.1908057(2003c:37063)

[16] F. John and L. Nirenberg, On functions of bounded mean oscillation, Comm. Pure Appl. Math. 14 (1961), 415-426. MR0131498 (24:A1348)

[17] P. W. Jones, Rectifiable sets and the traveling salesman problem, Invent. Math. 102 (1990), no. 1, 1-15. MR.1069238 (91i:26016)

[18] I. Karatzas and S. Shreve, Brownian motion and stochastic calculus, Springer-Verlag, Berlin, 1991. MR1121940 (92h:60127)

[19] G. F. Lawler, The dimension of the frontier of planar Brownian motion, Electron. Comm. Probab. 1 (1996), 29-47. MR1386292 (97g:60110)

[20] — Conformally invariant processes in the plane, Mathematical Surveys and Monographs, vol. 114, Amer. Math. Soc., Providence, 2005. MR2129588(2006i:60003)

[21] G. F. Lawler, O. Schramm, and W. Werner, The dimension of the Brownian frontier is 4/3, Math. Res. Lett. 8 (2001), no. 4, 401-411. MR1849257(2003a:60127b)

[22] _ Values of Brownian intersection exponents I: Half plane exponents, Acta Math. 187 (2001), no. 2, 237-273. MR.1879850 (2002m:60159a)

[23] (2001), no. 2, 275-308. MR1879851 (2002m:60159b)

[24] _ Analyticity of intersection exponents for planar Brownian motion, Acta Math. 189 (2002), no. 2, 179-201. MR1961197 (2003m:60231)

[25] _ Values of Brownian intersection exponents III: Two sided exponents, Ann. Inst. H. Poincaré Probab. Statist. 38 (2002), no. 1, 109-123. MR1899232 (2003d:60163)

[26] _ Conformal invariance of planar loop-erased random walks and uniform spanning trees, Ann. Probab. 32 (2004), no. 1B, 939-995. MR2044671 (2005f:82043) 
[27] - On the scaling limit of planar self-avoiding walk, Fractal geometry and applications: a jubilee of Benoît Mandelbrot, Part 2: Multifractals, probability and statistical mechanics, applications (Providence), Proc. Sympos. Pure Math., vol. 72, Amer. Math. Soc., 2004, pp. 339-364. MR2112127 (2006d:82033)

[28] J. Lind, Hölder regularity for the SLE trace, preprint (2005).

[29] Ch. Pommerenke, Boundary behaviour of conformal maps, Springer-Verlag, Berlin, 1992. MR:1217706 (95b:30008)

[30] J. Ratcliffe, Foundations of hyperbolic manifolds, Graduate Texts in Mathematics, vol. 149, Springer-Verlag, New York, 1994. MR.1299730 (95j:57011)

[31] S. Rohde and O. Schramm, Basic properties of SLE, Ann. of Math. 161 (2005), no. 2, 883924. MR2153402 (2006f:60093)

[32] B. Schmuland and W. Sun, A central limit theorem and law of the iterated logarithm for a random field with exponential decay of correlations, Canad. J. Math. 56 (2004), no. 1, 209-224. MR2031129 (2005f:60122)

[33] O. Schramm, Scaling limits of loop-erased random walks and uniform spanning trees, Israel J. Math. 118 (2000), 221-288. MR1776084 (2001m:60227)

[34] O. Schramm and S. Sheffield, Harmonic explorer and its convergence to SLE 4 , Ann. Probab. 33 (2005), no. 6, 2127-2148. MR2184093 (2006i:60013)

[35] S. Smirnov, Critical percolation in the plane: conformal invariance, Cardy's formula, scaling limits, C. R. Acad. Sci. Paris Sér. I. Math. 333 (2001), no. 3, 239-244. MR1851632 (2002f:60193)

[36] E. M. Stein, Harmonic analysis: real-variable methods, orthogonality, and oscillatory integrals, Princeton University Press, Princeton, 1993. MR 1232192 (95c:42002)

[37] W. Werner, Random planar curves and Schramm-Loewner evolutions, Lectures on probability theory and statistics: Ecole d'Eté de Probabilités de Saint-Flour XXXII-2002, Lecture Notes in Mathematics, vol. 1840, Springer-Verlag, Berlin, 2004, pp. 107-195. MR2079672 (2005m:60020)

Department of Mathematics, Massachusetts institute of Technology, Cambridge, Massachusetts 02139

E-mail address: kang@math.mit.edu 